\title{
Fluid-structure interaction problems with strong added-mass effect
}

\author{
S. R. Idelsohn ${ }^{1, *, \dagger, \ddagger}$, F. Del Pin ${ }^{2}$, R. Rossi ${ }^{1}$ and E. Oñate ${ }^{1}$ \\ ${ }^{1}$ International Center for Numerical Methods in Engineering (CIMNE), Universitat Politécnica de Catalunya \\ (UPC), Gran Capitan s/n, Barcelona, Spain \\ ${ }^{2}$ Livermore Software Technology Corporation, 7374 Las Positas Rd., Livermore, 94551 CA, U.S.A.
}

\begin{abstract}
SUMMARY
In this paper, the so-called added-mass effect is investigated from a different point of view of previous publications. The monolithic fluid-structure problem is partitioned using a static condensation of the velocity terms. Following this procedure the classical stabilized projection method for incompressible fluid flows is introduced. The procedure allows obtaining a new pressure segregated scheme for fluid-structure interaction problems, which has good convergent characteristics even for biomechanical application, where the added-mass effect is strong. The procedure reveals its power when it is shown that the same projection technique must be implemented in staggered FSI methods. Copyright (c) 2009 John Wiley \& Sons, Ltd.
\end{abstract}

Received 22 July 2008; Accepted 1 May 2009

KEY WORDS: added-mass effect; fluid-structure interactions; FSI; pressure segregation

\section{INTRODUCTION}

Fluid-structure interaction problems involving an incompressible viscous flow and elastic nonlinear structure have been solved in the past using different methods: Partitioned (or staggered) [1-4] approaches are probably the most popular solution techniques for the simulation of coupled problems as they allow using specifically designed codes on the different domains and offer significant benefits in terms of efficiency: smaller and better conditioned subsystems are solved instead of a single problem. Loosely (or weakly) [5] and strongly coupled [6-12] schemes are distinguished in the partitioned case: loosely coupling schemes require only one solution of either field per time step in a sequentially staggered manner and are thus particularly appealing in terms of efficiency. Strongly coupled schemes give, after an iterative process, the same results as non-partitioned (also named monolithic) algorithms. Both, the strongly coupled as well as the

\footnotetext{
*Correspondence to: S. R. Idelsohn, International Center for Numerical Methods in Engineering (CIMNE), Universitat Politécnica de Catalunya (UPC), Gran Capitan s/n, Barcelona, Spain.

${ }^{\dagger}$ E-mail: sergio@cimne.upc.edu

${ }^{\ddagger}$ ICREA Research Professor at CIMNE.

Copyright (C) 2009 John Wiley \& Sons, Ltd.
} 
monolithic scheme, lead to expensive simulations since, at each time step, a sub-iteration algorithm including the fluid and the structure domains has to be performed in the partitioned strongly coupled scheme. Alternatively, fully coupled systems including the equations for the fluid and the structure must be solved for the monolithic procedure.

There is a key difference between the strongly coupled scheme and the monolithic scheme: the iterative process in the strongly coupled scheme may be difficult (even non-convergent) when the so-called 'added-mass effect' is important $[13,14]$. Indeed, in such situation, a monolithic scheme seems to be necessary to avoid numerical instabilities. In fluid mechanics, added mass or virtual mass is the inertia added to a system because an accelerating or decelerating body must move some volume of surrounding fluid as it moves through it, since the object and fluid cannot occupy the same physical space simultaneously.

However, the name 'added-mass effect' has been used in the literature to indicate the numerical instabilities that typically occur in the internal flow of an incompressible fluid whose density is close to the structure density. We will use the same terminology to be consistent with previous papers, but as will be shown later, the instabilities are not necessarily caused by a fluid density close to the structure density. There are other reasons, as the elasticity coefficients and time step size that must be taken into account to avoid unstable solutions. The added-mass effect does not occur in aero elasticity problems, but it becomes very important in biomechanics applications where the materials are normally muscles and arteries and the fluid is blood.

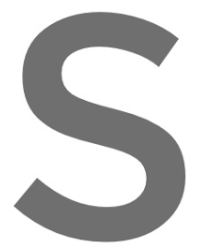

\section{Weakly coupled sqhemes a this effect is significant \\ There is a third situa the monolithic solution from the displacement or the velocity field. In} coupled way the fluid and the solid equations,
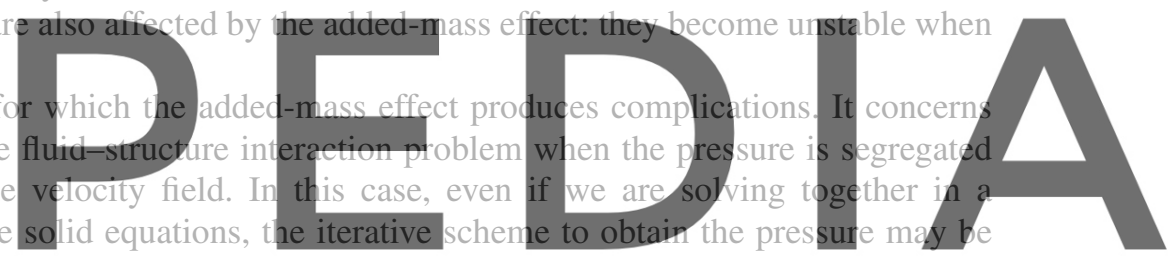
difficult and even non-convergent.

\section{Register for free at huttps//WWW.Scipedia.com to download the version without the watermark \\ 1. Instabilities in the loosely coupled scheme. \\ 2. Non-convergent iterative solutions in the strongly coupled scheme.}

3. Non-convergent iterative solutions in the monolithic scheme with pressure segregation.

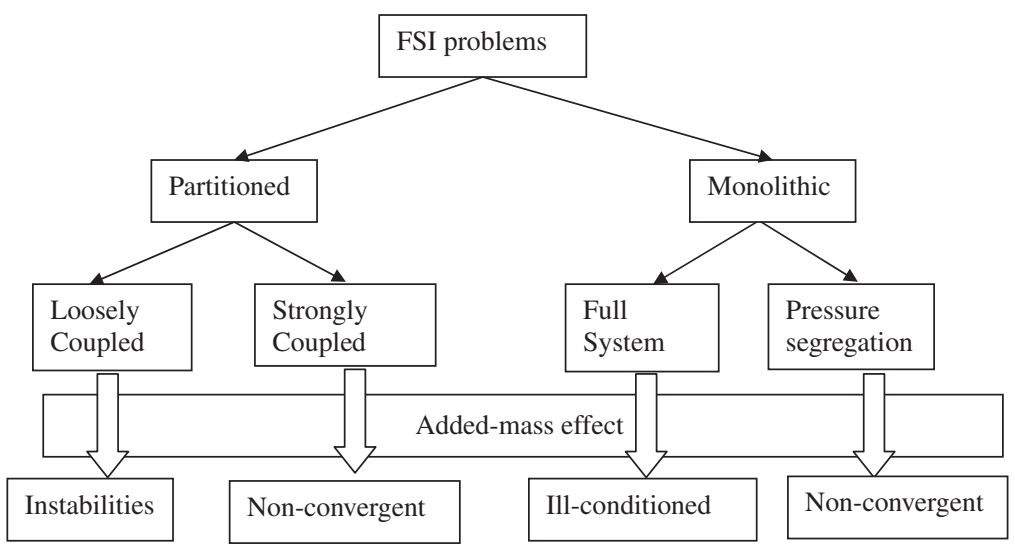

Figure 1. Summary of the numerical troubles induced by the added-mass effect when solving FSI problems. 
Such monolithic schemes with pressure segregation are first addressed in [15]. A further development of the idea is presented in [14]. Exploiting the unified formulation some mathematical explanation for the so-called added-mass effect was presented in [13]. The role played by the stabilization in the field was highlighted in a very clear way in [16]. An alternative coupling paradigm based on the so-called Robin-Robin condition is presented in [17], while a different formulation based on the use of a modified projection at the interface is presented in [12].

The main idea of this article is totally different than the previously reported references. First, we put in evidence that the numerical instabilities that appear with the added-mass effect is a consequence of the pressure segregation (case 3 in the previous list) and that a correct understanding of the pressure segregation effect yields different solutions to the added-mass problem, which may be successfully applied to cases 1 and 2 . Starting from the monolithical approach the 'exact' way to segregate the pressure (in fact, it is only exact for the linear case) is described. This exact way is represented by a tangent matrix that, for the linear case, gives the result in only one iteration. An approximation to this tangent matrix is then proposed. Several other approximations may also be thought with the same idea, but the one proposed here shows excellent convergence rates for strong added-mass effect problems. The achieved formulation is independent of the time integration scheme and may be applied to fully implicit, semi implicit or explicit time integrations.

In the following section we will study the numerical solution of the monolithic scheme with pressure segregation. At this point we can ask ourselves why we are trying to segregate the pressure

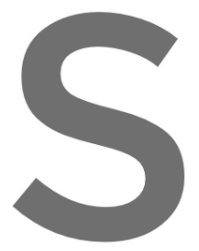
from the rest of the

(1) Because to solve the pressure together with the rest of the and/or displa system to be

(2) Because partition when the structural dontin is solved, the
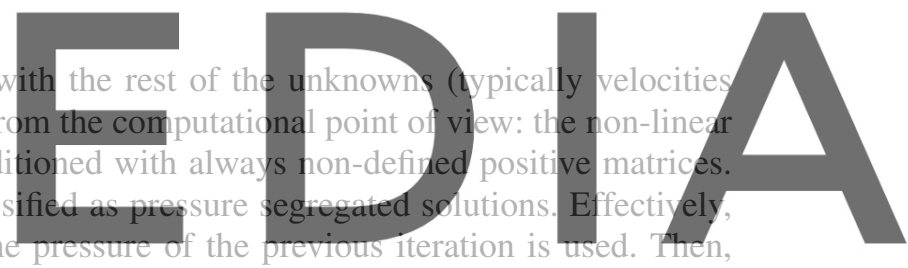
if a procedure is unstable using a monolithic algorithm with pressure segregation, it will be

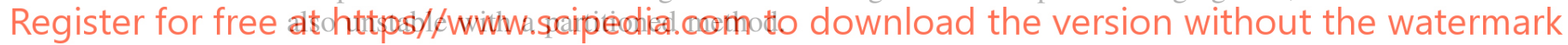

The segregation of the pressure can be conveniently performed using a Chorin-Temam projection scheme [18-20]. This splitting procedure works convenientiy for incompressibie flows. Nevertheless, we will introduce pressure segregation via a simple static condensation procedure. This static condensation will explain the Chorin-Teman projection as a particular case and will allow generalizing the Chorin-Temam scheme for fluid-structure interaction problems.

\section{THE DISCRETIZED EQUATIONS TO BE SOLVED IN AN FSI PROBLEM}

The equations to be solved for both the incompressible fluid and the elastic solid domains are the momentum equations:

$$
\rho a_{i}=\rho \frac{D V_{i}}{D t}=\frac{\partial \sigma_{i j}}{\partial x_{j}}+\rho f_{i}
$$

where $\sigma_{i j}$ is the Cauchy stress tensor, $\rho$ the density, $a_{i}$ the acceleration vector equal to the total derivative of the velocity $V_{i}$ and $f_{i}$ a body force vector. 
In the incompressible part of the domain, mass conservation equation must be enforced:

$$
\varepsilon_{V}=\frac{\partial V_{i}}{\partial x_{i}}=0
$$

The boundary conditions for both domains are:

$$
\sigma_{n i}=\overline{\sigma_{n i}} \text { in } \Gamma_{\sigma} \quad \text { and } \quad U_{i}=\overline{U_{i}} \text { in } \Gamma_{U}
$$

On the fluid domain, it is sometimes useful to use a moving framework different than the particle displacement. In this case, the acceleration vector may be written as a function of the framework velocity $V_{M j}$ as

$$
\frac{D V_{i}}{D t}=\frac{D F V_{i}}{D t}+\left(V_{j}-V_{M j}\right) \frac{\partial V_{i}}{\partial x_{j}}
$$

\section{where $D_{F} V_{i} / D t$ represents the framework acceleration.}

Apart from the incompressible condition the only difference between the fluid and the solid are the constitutive equations. Classical Newtonian constitutive equations for the fluid are expressed as a function of the rate of deformations and the pressure:
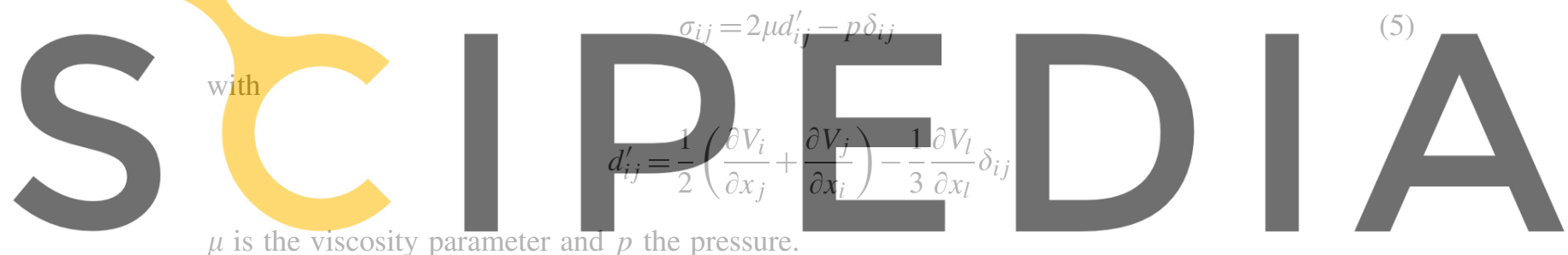

On the other hand, the constitutive equations for an elastic solid are written as the function of Register for theeseat: https//www.scipedia.com to download the version without the watermark

$$
\sigma_{i j}=C_{i j}^{k l} \varepsilon_{k i} \quad \text { with } \varepsilon_{i j}=\frac{1}{2}\left(\frac{\partial U_{i}}{\partial x_{j}}+\frac{\partial U_{j}}{\partial x_{i}}+\frac{\partial U_{i}}{\partial x_{j}} \frac{\partial U_{j}}{\partial x_{i}}\right)
$$

Nevertheless, once the time integration scheme has been chosen, both constitutive equations may be written as a function of the displacement rates or the velocities rates (adding always the pressure in the incompressible part).

Assuming for simplicity that an implicit Euler time integration has been chosen, then

$$
U_{i}^{n+1}=\Delta t\left(\theta V_{i}^{n+1}+(1-\theta) V_{i}^{n}\right)
$$

where the upper index indicates the time position, $\Delta t$ is the time step and $\theta$ is an integration parameter between 0 and 1 . To simplify the notation, in the following the upper index $n+1$ will be omitted.

For instance for a hypo elastic solid, the constitutive equation becomes:

$$
\sigma_{i j}=\hat{\sigma}_{i j}^{n}+\frac{2 \Delta t G}{J} d_{i j}^{\prime}+\frac{\Delta t}{J}\left(\lambda+\frac{2 G}{3}\right) \varepsilon_{V} \delta_{i j}
$$

where $\lambda$ and $G$ are the Lamé parameters, $J$ the Jacobian matrix and $\hat{\sigma}_{i j}^{n}$ the initial stress tensor. 
In the following and without loss of generality, we will consider that the constitutive equations for the solid and the fluid domains are expressed as a function of the velocity field (plus the pressure in the incompressible region). The same results and conclusions may be obtained using the displacement field as the main unknown.

Finally, in spite that the momentum equations for both domains, the solid and the fluid, are geometrically non-linear, we will consider, for simplicity in the theoretical aspect of this development, only the linear term, assuming that the matrices obtained are an approximation to the exact non-linear ones.

The weighted residual form of the momentum and mass conservation equations are:

$$
\int_{V} W_{l}\left(\rho \frac{D V_{i}}{D t}-\frac{\partial \sigma_{i j}}{\partial x_{j}}-\rho f_{i}\right) d V+\int_{\Gamma_{\sigma}} W_{l}\left(\sigma_{n i}-\overline{\sigma_{n i}}\right) d \Gamma=0
$$

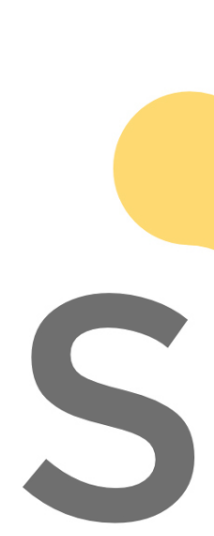

$$
\int_{V} W_{p}\left(-\varepsilon_{V}\right) \mathrm{d} V=0
$$

\section{and the weak form is:}

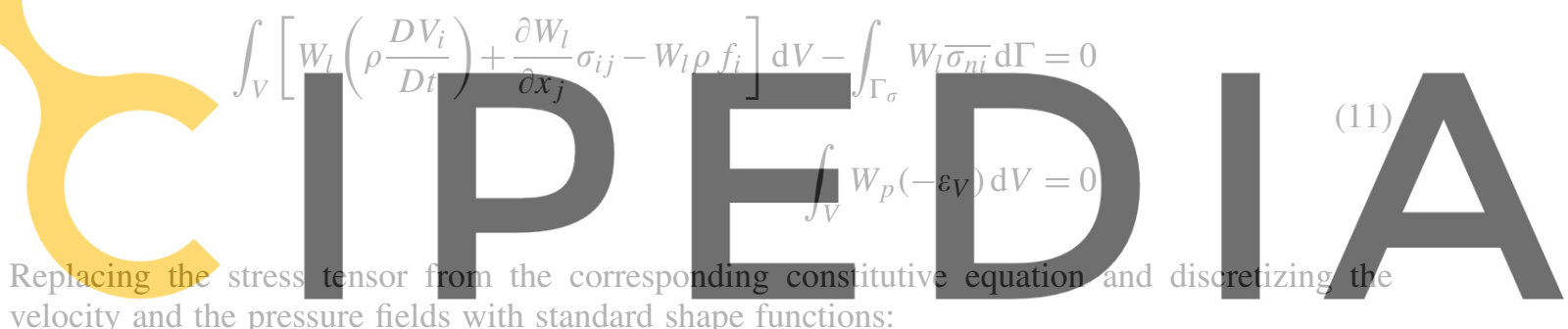

velocity and the pressure fields with standard shape functions:

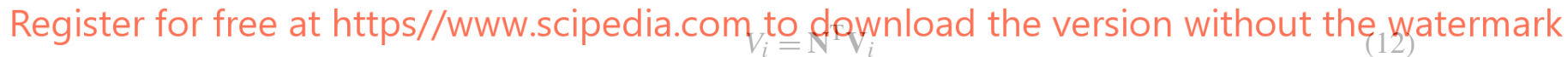

$$
p=\mathbf{N}_{p}^{\mathrm{T}} \mathbf{P}
$$

and using Galerkin weighting functions:

$$
\left[\mathbf{W}_{1}, \mathbf{W}_{2}, \mathbf{W}_{3}\right]=\left[\begin{array}{ccc}
\mathbf{N} & 0 & 0 \\
0 & \mathbf{N} & 0 \\
0 & 0 & \mathbf{N}
\end{array}\right], \quad \mathbf{W}_{p}=\mathbf{N}_{p}
$$

the global fluid-structure interaction problem may be written in a compact monolithic form as:

$$
\left[\begin{array}{cc}
\left(\frac{\mathbf{M}_{\rho}}{\Delta t}+\mathbf{K}\right) & -\mathbf{B} \\
-\mathbf{B}^{\mathrm{T}} & 0
\end{array}\right]\left[\begin{array}{l}
\mathbf{V} \\
\mathbf{P}
\end{array}\right]=\left[\begin{array}{c}
\mathbf{F}+\frac{\mathbf{M}_{\rho}}{\Delta t} \mathbf{V}^{n} \\
0
\end{array}\right]
$$


where $\mathbf{M}_{\rho}$ is the mass matrix that is a function of the fluid density $\rho_{\mathrm{f}}$ or the solid density $\rho_{\mathrm{s}}$ and the shape functions:

$$
\mathbf{M}_{\rho}=\left[\begin{array}{ccc}
\mathbf{M}_{11} & 0 & 0 \\
0 & \mathbf{M}_{22} & 0 \\
0 & 0 & \mathbf{M}_{33}
\end{array}\right]
$$

with $\mathbf{M}_{i i}=\int_{V} \mathbf{N} \rho \mathbf{N}^{\mathrm{T}} \mathrm{d} V$.

$\mathbf{K}$ is the stiffness matrix function depending on the viscosity $\mu$ in the fluid part of the Lamé parameters $(G \Delta t)$ and $(\lambda \Delta t)$ in the solid part and the derivatives of the shape functions

$$
\mathbb{K}=\left[\begin{array}{lll}
\mathbb{K}_{11} & \mathbb{K}_{21} & \mathbb{K}_{31} \\
\mathbb{K}_{12} & \mathbb{K}_{22} & \mathbb{K}_{32} \\
\mathbb{K}_{13} & \mathbb{K}_{23} & \mathbb{K}_{33}
\end{array}\right]
$$

\section{with $\mathbf{K}_{i j}=\mathbf{K}_{i j}^{1}+\mathbf{K}_{i j}^{2}+\mathbf{K}_{i j}^{3}$. In the fluid part:}
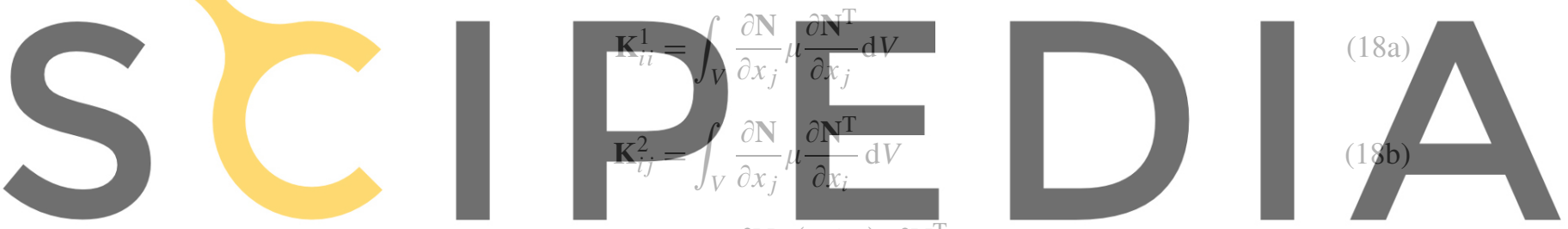

Register for free at https//www.scipedia.com tioldownlöad the version without the watermark

and in the solid domain:

$$
\begin{aligned}
& \mathbf{K}_{i i}^{1}=\int_{V} \frac{\partial \mathbf{N}}{\partial x_{j}}\left(\frac{\Delta t G}{J}\right) \frac{\partial \mathbf{N}^{\mathrm{T}}}{\partial x_{j}} \mathrm{~d} V \\
& \mathbf{K}_{i j}^{2}=\int_{V} \frac{\partial \mathbf{N}}{\partial x_{j}}\left(\frac{\Delta t G}{J}\right) \frac{\partial \mathbf{N}^{\mathrm{T}}}{\partial x_{i}} \mathrm{~d} V \\
& \mathbf{K}_{i j}^{3}=\int_{V} \frac{\partial \mathbf{N}}{\partial x_{i}}\left(\frac{\Delta t \lambda}{J}\right) \frac{\partial \mathbf{N}^{\mathrm{T}}}{\partial x_{j}} \mathrm{~d} V
\end{aligned}
$$

If the displacements of the moving framework are different than the particle displacements, matrix $\mathbf{K}$ includes the convective terms $\mathbf{K}_{i j}^{4}$ :

$$
\mathbf{K}_{i i}^{4}=\int_{V} \mathbf{N}\left(V_{j}-V_{M j}\right) \frac{\partial \mathbf{N}^{\mathrm{T}}}{\partial x_{j}} \mathrm{~d} V
$$

Matrix $\mathbf{B}$ affects the incompressible part of the domain. This means that $\mathbf{B}$ has non-zero terms only in the degrees of freedom (DOF) related with the fluid including the solid-fluid interfaces. 
The form of matrix $\mathbf{B}$ is:

$$
\mathbf{B}^{\mathrm{T}}=\left[\mathbf{B}_{1}^{\mathrm{T}}, \mathbf{B}_{2}^{\mathrm{T}}, \mathbf{B}_{3}^{\mathrm{T}}\right] \quad \text { with } \mathbf{B}_{i}^{\mathrm{T}}=\int_{V} \mathbf{N}_{p} \frac{\partial \mathbf{N}^{\mathrm{T}}}{\partial x_{i}} \mathrm{~d} V
$$

Equation (15) represents the coupled monolithic fluid-structure interaction problem that must be solved. It is well known that this system of equations must be stabilized for some class of equal order interpolations (e.g. when $\mathbf{N}_{p}=\mathbf{N}$ ) [21-23].

Independent of the method chosen to stabilize the problem, we will assume that the problem has been conveniently stabilized by a matrix $\mathbf{S}$ (to be defined later on Section 3.3) in such way that the problem reads:

$$
\left[\begin{array}{cc}
\left(\begin{array}{c}
\frac{\mathbf{M}_{\rho}}{\Delta t}+\mathbb{K} \\
-\mathbb{B}^{\mathrm{T}}
\end{array}\right. & -\mathbb{B}
\end{array}\right]\left[\begin{array}{c}
\mathbf{V} \\
\mathbb{P}
\end{array}\right]=\left[\begin{array}{c}
\mathbb{F}+\frac{\mathbf{M}_{\rho}}{\Delta t} \mathbf{V}^{n} \\
0
\end{array}\right]
$$

The stabilization terms introduce an error in the original system (15). In particular, the incompressibility condition originally discretized by (see Equation (15)):

$$
\mathbf{B}_{i}^{\mathrm{T}} \mathbf{V}_{i}=\mathbf{0}
$$
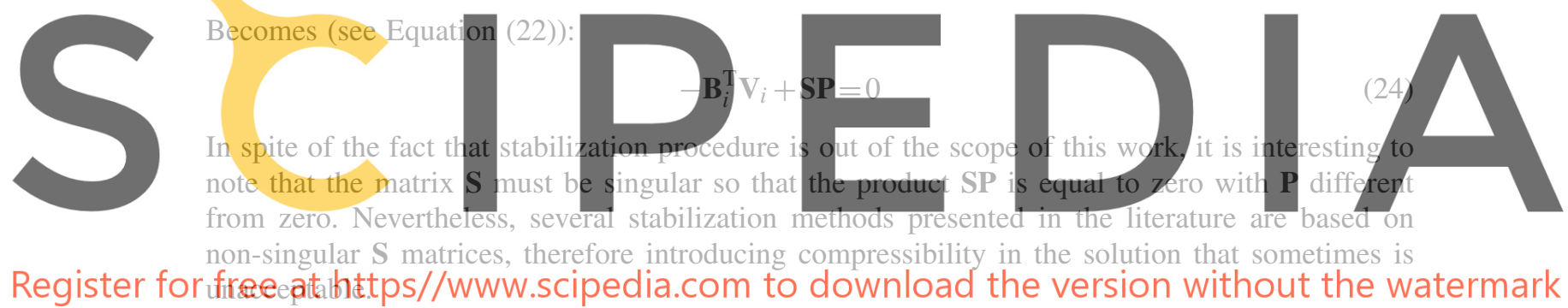

\section{MONOLITHIC SOLUTION OF THE FSI EQUATIONS BY PRESSURE SEGREGATION}

Solution of Equation (15) or their analogous stabilized version (22) as a fully coupled system is sometimes expensive due to ill-conditioning problems. A more convenient way to solve that system is segregating the pressure from the remaining unknowns (in our examples the velocity field). Segregation means to separate during the solution process the pressure from the velocity variables in a staggered way: first the velocities (or the pressure) are evaluated independent of the pressure (or the velocities) and then the solution for the pressure (or the velocities) is found using the previous results. Segregation of the pressure has several advantages as:

(1) Decreases the number of DOF to be solved simultaneously.

(2) Avoids ill-conditioned matrices.

(3) Allows using unified formulations for the fluid and solid domains.

(4) It separates the velocity components.

(5) It separates the non-linearity.

(6) Allows us to draw some conclusions to be used in partitioned schemes (for which the pressure is always segregated from the solid part). 
There are several ways to segregate the pressure from the velocity. The simplest one is to assume an initial value for the pressure, compute the velocities using this initial value and then evaluate the pressure iteratively. A more sophisticated scheme to segregate the pressure is the Chorin-Temam projection scheme [18-20], which will be discussed later.

In order to simplify the discussion, the following change of variable will be introduced: being $\mathbf{P}_{0}$ any arbitrary vector of the same dimension of the pressure, we define the following new unknown:

$$
\boldsymbol{\delta P}=\mathbf{P}-\mathbf{P}_{0}
$$

Note that $\mathbf{P}_{0}$ is not necessarily the initial pressure vector at time $t=0$. It is any arbitrary vector.

The system of equations to be solved becomes:

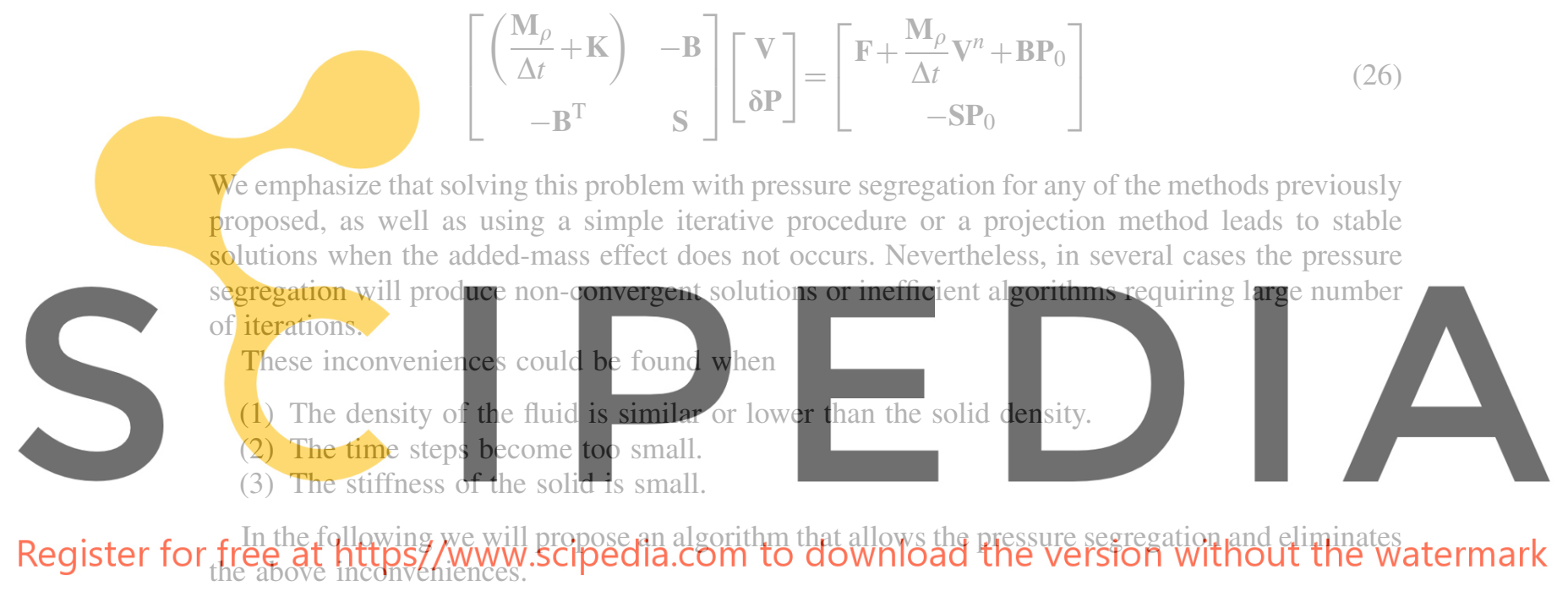

\subsection{Static condensation of the pressure}

The more precise way to segregate the pressure from the velocity is via static condensation (it is exact in case of linear system of equations). Static condensation is a procedure to solve a system of equations in a partitioned way. It consists of inverting a part of the initial matrix. For instance, in system (26) we can condensate the pressure by inverting matrix $\mathbf{S}$, or condensate the velocity by inverting matrix $\left(\left(\mathbf{M}_{\rho} / \Delta t\right)+\mathbf{K}\right)$. As stated before, matrix $\mathbf{S}$ must be singular and then the only possibility is to condense the velocity field.

From the first row of (26) the velocity field may be obtained as:

$$
\mathbf{V}=\left(\frac{\mathbf{M}_{\rho}}{\Delta t}+\mathbf{K}\right)^{-1}\left(\mathbf{F}+\frac{\mathbf{M}_{\rho}}{\Delta t} \mathbf{V}^{n}+\mathbf{B} \mathbf{P}_{0}+\mathbf{B} \delta \mathbf{P}\right)
$$

Inserting this into the second line of Equation (26) gives:

$$
-\mathbf{B}^{\mathrm{T}}\left(\frac{\mathbf{M}_{\rho}}{\Delta t}+\mathbf{K}\right)^{-1}\left(\mathbf{F}+\frac{\mathbf{M}_{\rho}}{\Delta t} \mathbf{V}^{n}+\mathbf{B} \mathbf{P}_{0}+\mathbf{B} \delta \mathbf{P}\right)+\mathbf{S} \delta \mathbf{P}=-\mathbf{S P}_{0}
$$


This means that the static condensation of the velocity allows one to solve the problem in two steps:

(I)

$$
\left[-\mathbf{B}^{\mathrm{T}}\left(\frac{\mathbf{M}_{\rho}}{\Delta t}+\mathbf{K}\right)^{-1} \mathbf{B}+\mathbf{S}\right] \boldsymbol{\delta} \mathbf{P}=\mathbf{B}^{\mathrm{T}}\left(\frac{\mathbf{M}_{\rho}}{\Delta t}+\mathbf{K}\right)^{-1}\left(\mathbf{F}+\frac{\mathbf{M}_{\rho}}{\Delta t} \mathbf{V}^{n}+\mathbf{B} \mathbf{P}_{0}\right)-\mathbf{S} \mathbf{P}_{0}
$$

(II)

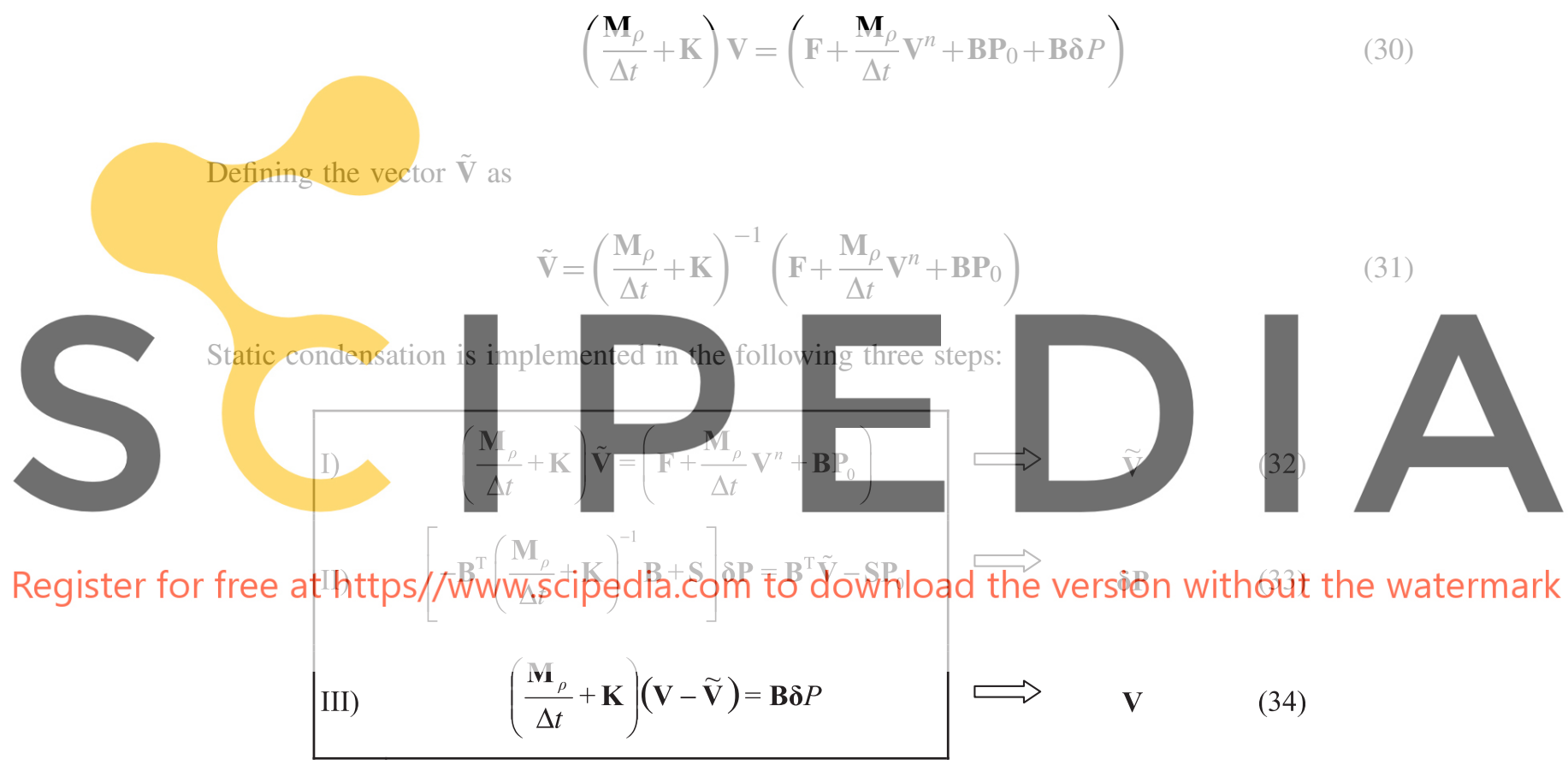

Equations (32)-(34) represent the way to segregate the pressure from the velocity in an exact way. It is a very expensive procedure from the computational point of view, but if enough resources are available it is the correct method to apply. On the other hand, Equations (32)-(34) suggest a procedure to approximate the exact algorithm and to obtain a more efficient way to segregate the pressure.

It is interesting to note the analogy between Equations (32)-(34) with the classical Chorin-Teman projection scheme. Effectively, the first Equation (32) is the evaluation of the compressible velocity $\tilde{\mathbf{V}}$. Using $\mathbf{P}_{0}=\mathbf{0}$ the first-order scheme is obtained. Using $\mathbf{P}_{0}=\mathbf{P}^{n}$ second-order approximation may be developed. Equation (33) is in fact the exact equation corresponding to the Laplace equation in a projection method and (34) is the exact equation to obtain the incompressible velocity as a function of the pressure gradients. Then we can say that Chorin-Teman projection method is an approximation to the exact static condensation of the velocity field. In the following section we present other approximations more suitable for FSI problems. 


\subsection{Approximation to the static condensation}

The Chorin-Teman projection method [20] is equivalent to approximate the matrix $\mathbf{B}^{\mathrm{T}}\left(\left(\mathbf{M}_{\rho} /\right.\right.$ $\Delta t)+\mathbf{K})^{-1} \mathbf{B}$ in Equation (33) by the Laplace matrix $L$ :

$$
\mathbf{B}^{\mathrm{T}}\left(\frac{\mathbf{M}_{\rho}}{\Delta t}+\mathbf{K}\right)^{-1} \mathbf{B} \cong \frac{\Delta t}{\rho} \mathbf{L}
$$

where:

$$
\mathbf{L}=\int_{V} \frac{\partial \mathbf{N}}{\partial x_{j}} \frac{\partial \mathbf{N}^{\mathrm{T}}}{\partial x_{j}} \mathrm{~d} V
$$

This approximation is acceptable for non-viscous or nearly inviscid flows for which matrix $\mathbb{K}$ is negligible versus the mass matrix $\mathbf{M}_{\rho} / \Delta t$. The remaining matrix $\mathbf{B}^{\mathrm{T}}\left(\mathbf{M}_{\rho} / \Delta t\right)^{-1} \mathbf{B}$ is approximately equal to $(\Delta t / \rho) \mathbf{L}$ (see the definition of $\mathbf{B}, \mathbf{M}_{\rho}$ and $\mathbf{L}$ ) for a lumped mass matrix and continuous pressure shape functions.

A more suitable way to approximate matrix $\mathbf{B}^{\mathrm{T}}\left(\left(\mathbf{M}_{\rho} / \Delta t\right)+\mathbf{K}\right)^{-1} \mathbf{B}$ for cases where matrix $\mathbf{K}$ is not negligible will be proposed next.

Being $\mathbf{M}=\sum \mathbf{M}^{e}$ and $\mathbf{K}=\sum \mathbb{K}^{e}$ where $\mathbf{M}^{e}$ and $\mathbb{K}^{e}$ are the mass and stiffness matrices of an element ' $\mathrm{e}$ '. Let us
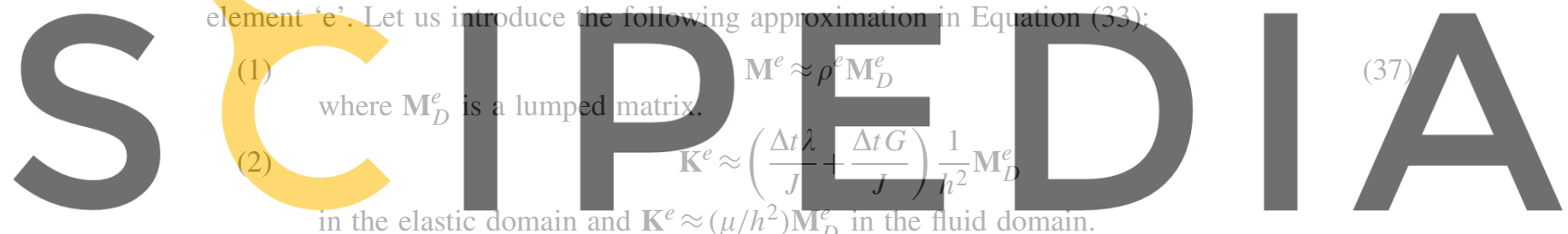

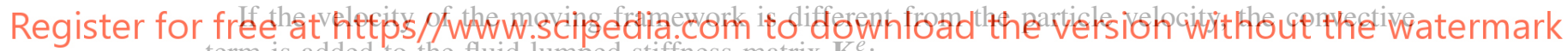
term is added to the fluid lumped stiffness matrix $\mathbf{K}^{e}$ :

(2bis)

$$
\mathbf{K}^{e} \approx\left(\frac{\mu}{h^{2}}+\frac{\left|\mathbf{V}-\mathbf{V}_{m}\right|}{h}\right) \mathbf{M}_{D}^{e}
$$

In all previous definitions, $h$ represents a characteristic element size (for instance, the average distance between the element nodes).

Note that previous approximations are also used by other authors in related fields. See, for example, [24].

It must be noted that using the same idea, different possibilities for the lumped mass and stiffness matrices may be proposed. In (37), (38) just the diagonal terms of each matrix are chosen, but other most sophisticated lumped matrices may be used. We emphasize that these lumped matrices are used exclusively in the pressure Equation (33). In Equations (32) and (34) the fully consistent matrices are used.

With the previous approximations, the original matrix in Equation (33) becomes:

$$
\left(\frac{\mathbf{M}}{\Delta t}+\mathbf{K}\right)^{-1}=\sum\left(\frac{\mathbf{M}^{e}}{\Delta t}+\mathbf{K}^{e}\right)^{-1} \approx \sum\left(\tau^{e}\left[\mathbf{M}^{e}\right]_{D}^{-1}\right)=\mathbf{M}_{T}^{-1}
$$


where $\mathbf{M}_{T}^{-1}$ is a diagonal matrix obtained from the assembly of the element contributions $\tau^{e}\left[\mathbf{M}^{e}\right]_{D}^{-1}$ with

$$
\tau^{e}=\left(\frac{\rho_{s}}{\Delta t}+\frac{\Delta t \lambda}{J h^{2}}+\frac{\Delta t G}{J h^{2}}\right)^{-1}
$$

in the elastic domain and

$$
\tau^{e}=\left(\frac{\rho_{f}}{\Delta t}+\frac{\mu}{h^{2}}+\frac{\left|\mathbf{V}^{e}-\mathbf{V}_{m}^{e}\right|}{h}\right)^{-1}
$$

in the incompressible domain.

The first two approximations may be written as:

$$
\mathbf{B}^{\mathrm{T}}\left(\frac{\mathbf{M}_{\rho}}{\Delta t}+\mathbf{K}\right)^{-1} \mathbf{B} \approx \mathbf{B}^{\mathrm{T}} \mathbf{M}_{T}^{-1} \mathbf{B}
$$

Finally, a third approximation similar to the classical one introduced in projection methods is added:

$$
\mathbf{B}^{\mathrm{T}} \mathbf{M}_{T}^{-1} \mathbf{B} \approx \mathbf{L}_{\tau}
$$

where $\mathbf{L}_{\tau}=\sum\left(\tau^{e} \mathbf{L}^{e}\right)$, being $\mathbf{L}^{e}$ the Laplace matrix for the element.

Then, the three steps algorithm reads:

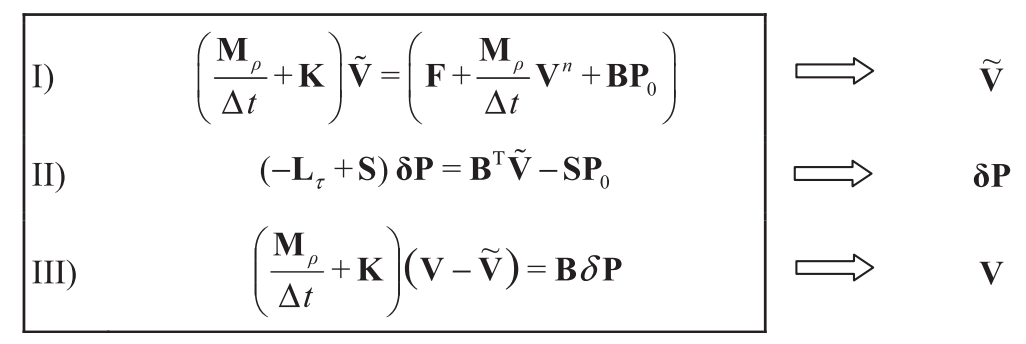

Of course, Equation (45) is an approximation to Equation (33) when $\mathbf{B}^{\mathrm{T}}\left(\left(\mathbf{M}_{\rho} / \Delta t\right)+\mathbf{K}\right)^{-1} \mathbf{B}$ is replaced by $\mathbf{L}_{\tau}$. This approximation introduces an error in the evaluation of $\mathbf{P}$. In order to diminish this error, an iterative procedure may be used to approximate $\mathbf{P}_{0}$ by $\mathbf{P}$. Effectively, now the introduction of the arbitrary variable $\mathbf{P}_{0}$ in Equation (25) becomes justifiable. Hence, once $\mathbf{P}$ is evaluated at the $k$ iteration, then the next iteration is started with $\mathbf{P}_{0}=\mathbf{P}^{k}$ in Equation (44). Note that the error introduced by the approximation to $\mathbf{B}^{\mathrm{T}}\left(\left(\mathbf{M}_{\rho} / \Delta t\right)+\mathbf{K}\right)^{-1} \mathbf{B}$ becomes negligible when $\boldsymbol{\delta} \mathbf{P} \rightarrow 0$.

Another way to introduce the previous approximation is by keeping $\mathbf{P}$ in the r.h.s. of Equation (45). The system of equations to be solved is now:

$$
\begin{aligned}
& \left(\frac{\mathbf{M}_{\rho}}{\Delta t}+\mathbf{K}\right) \tilde{\mathbf{V}}=\left(\mathbf{F}+\frac{\mathbf{M}_{\rho}}{\Delta t} \mathbf{V}^{n}+\mathbf{B} \mathbf{P}_{0}\right) \quad \rightleftarrows \quad \tilde{\mathbf{V}} \\
& \left(-\mathbf{L}_{\tau}+\mathbf{S}\right) \mathbf{P}=\mathbf{B}^{\mathrm{T}} \tilde{\mathbf{V}}-\mathbf{B}^{\mathrm{T}}\left(\frac{\mathbf{M}_{\rho}}{\Delta t}+\mathbf{K}\right)^{-1} \mathbf{B} \mathbf{P}_{0} \quad \longleftrightarrow \quad \boldsymbol{\delta} \mathbf{P} \\
& \left(\frac{\mathbf{M}_{\rho}}{\Delta t}+\mathbf{K}\right)(\mathbf{V}-\tilde{\mathbf{V}})=\mathbf{B}\left(\mathbf{P}-\mathbf{P}_{0}\right) \quad \longrightarrow \quad \mathbf{V}
\end{aligned}
$$


In order to facilitate the solution of Equation (48), let us introduce the following auxiliary vector $\mathbf{T}_{0}$ :

$$
\mathbf{T}_{0}=\left(\frac{\mathbf{M}_{\rho}}{\Delta t}+\mathbf{K}\right)^{-1} \mathbf{B} \mathbf{P}_{0}
$$

Then the four steps algorithm reads:

$$
\begin{array}{lll}
\left(\frac{\mathbf{M}_{\rho}}{\Delta t}+\mathbf{K}\right) \tilde{\mathbf{V}}=\left(\mathbf{F}+\frac{\mathbf{M}_{\rho}}{\Delta t} \mathbf{V}^{n}+\mathbf{B} \mathbf{P}_{0}\right) & \longrightarrow & \mathbf{\mathbf { V }} \\
\left(\frac{\mathbf{M}_{\rho}}{\Delta t}+\mathbf{K}\right) \mathbf{T}_{0}=\mathbf{B} \mathbf{P}_{0} & & \\
\left(-\mathbf{L}_{\tau}+\mathbf{S}\right) \mathbf{P}=\mathbf{B}^{\mathrm{T}} \tilde{\mathbf{V}}-\mathbf{B}^{\mathrm{T}} \mathbf{T}_{0} & \longrightarrow & \mathbf{T}_{0} \\
\left(\frac{\mathbf{M}_{\rho}}{\Delta t}+\mathbf{K}\right)(\mathbf{V}-\tilde{\mathbf{V}})=\mathbf{B}\left(\mathbf{P}-\mathbf{P}_{0}\right) & \longrightarrow \mathbf{P}
\end{array}
$$

Remark: Equations (50)-(53) are conceptually different from Equations (44)-(46). In fact, in Equations (44)-(46) the error introduced by the approximation to the static condensation disappears when $\boldsymbol{\delta} \mathbf{P} \rightarrow \mathbf{0}$. However, this error does not disappear in Equations (50)-(53), remaining as a nonzero term even for $\boldsymbol{\delta P}=\mathbf{0}$. This remaining term introduces an additional stabilization in the pressure equation that, in certain cases, is enough to obtain stable solutions. However, this additional term does not always guarantee a stable solution and for this reason Equations (44)-(46) will be used in the following part of this work.

It is also interesting to note the similitude of Equations (50)-(53) to the classical projection methods (see for instance Codina [20]). Vector $\mathbf{T}_{0}$ represents the projection of the pressure gradients in the velocity field (see Equation (51)). In our approach, this method has been obtained in a more natural way as a consequence of static condensation. Equations (50)-(53) represent a generalization of the Chorin-Teman projection method to problems for which the $\mathbf{K}$ matrix is not negligible versus the $\mathbf{M}_{\rho} / \Delta t$ matrix.

\subsection{Definition of the stabilizing matrix}

As stated before, Equation (45) must be stabilized for equal order elements in order to overcome the BBL condition. Matrix $\mathbf{S}$ represents this stabilizing matrix in Equation (45).

The error introduced in approximating $\mathbf{B}^{\mathrm{T}}\left(\left(\mathbf{M}_{\rho} / \Delta t\right)+\mathbf{K}\right)^{-1} \mathbf{B}$ by $\mathbf{L}_{\tau}$ introduces a stabilization during the iterative process while $\boldsymbol{\delta} \mathbf{P} \neq 0$. Nevertheless, in order to have a more general stabilization algorithm independently of the iterative process, a standard projection method will be used to stabilize the pressure equation.

Projection methods are based on the difference between the pressure gradients (which are noncontinuous between elements) and a continuous function of the pressure gradients (the projection of the pressure gradients in the continuous velocity space).

Let us define the pressure gradient projections $\boldsymbol{\Pi}_{i}=\tau_{0}\left(\partial p / \partial x_{i}\right)$ where $\tau_{0}$ is a parameter that will be defined later on. A simple Galerkin weighting approximation gives:

$$
\int_{V} \mathbf{N} \tau_{0} \frac{\partial \mathbf{N}_{p}^{\mathrm{T}}}{\partial x_{i}} \mathrm{~d} V \mathbf{P}=\int_{V} \mathbf{N} \boldsymbol{\Pi}_{i} \mathrm{~d} V=\int_{V} \mathbf{N} \mathbf{N}^{\mathrm{T}} \mathrm{d} V \boldsymbol{\Pi}
$$


or

$$
\begin{aligned}
\int_{V} \mathbf{N} \frac{\partial \mathbf{N}_{p}^{\mathrm{T}}}{\partial x_{i}} \mathrm{~d} V \mathbf{P} & =\int_{V} \mathbf{N} \frac{1}{\tau_{0}} \mathbf{N}^{\mathrm{T}} \mathrm{d} V \boldsymbol{\Pi} \\
\mathrm{DP} & =\mathbf{M}_{\tau_{0}} \boldsymbol{\Pi} \Rightarrow \boldsymbol{\Pi}=\mathbf{M}_{\tau_{0}}^{-1} \mathbf{D P}
\end{aligned}
$$

with

$$
\mathbf{D}^{\mathrm{T}}=\left[\mathbf{D}_{1}^{\mathrm{T}}, \mathbf{D}_{2}^{\mathrm{T}}, \mathbf{D}_{3}^{\mathrm{T}}\right], \quad \mathbf{D}_{i}^{\mathrm{T}}=\int_{V} \frac{\partial \mathbf{N}}{\partial x_{i}} \mathbf{N}_{p}^{\mathrm{T}} \mathrm{d} V
$$

and

$$
\mathbf{M}_{\tau_{0}}=\left[\begin{array}{ccc}
\int_{V} \mathbf{N} \frac{1}{\tau_{0}} \mathbf{N}^{\mathrm{T}} \mathrm{d} V & 0 & 0 \\
0 & \int_{V} \mathbf{N} \frac{1}{\tau_{0}} \mathbf{N}^{\mathrm{T}} \mathrm{d} V & 0 \\
0 & 0 & \int_{V} \mathbf{N} \frac{1}{\tau_{0}} \mathbf{N}^{\mathrm{T}} \mathrm{d} V
\end{array}\right]
$$

The standard stabilization term [21,22] is defined by the divergence of the difference between the gradient and the projection gradient pressure:

$$
\mathbf{S P}=-\int_{V} \mathbf{N}_{p} \frac{\partial}{\partial x_{i}}\left(\boldsymbol{\Pi}_{i}-\tau_{0} \frac{\partial p}{\partial x_{i}}\right) \mathrm{d} V
$$

Parameter $\tau_{0}$ has been introduced in order to keep the consistence of the dimensions. Taking into account that $\mathbf{S P}$ will be added to the mass conservation equation, a correct value for $\tau_{0}$ is $\tau_{0}=\Delta t / \rho$.

It must be noted that $\tau_{0}$ changes for each element. This means that matrix $\mathbf{M}_{\tau_{0}}^{-1}$ must be built element by element using standard assembly process.

In a more general way, taking into account the 'natural' definition of $\tau$ for the static condensation of Equation (41), the following definition of $\tau_{0}^{e}$ is also useful:

$$
\tau_{0}^{e}=\tau^{e}=\left(\frac{\rho_{f}}{\Delta t}+\frac{\mu}{h^{2}}+\frac{\left|\mathbf{V}^{e}-\mathbf{V}_{m}^{e}\right|}{h}\right)^{-1}
$$

Integrating by parts Equation (58):

$$
\begin{aligned}
\mathbf{S P}= & -\int_{V} \mathbf{N}_{p} \frac{\partial}{\partial x_{i}}\left(\boldsymbol{\Pi}_{i}-\tau_{0} \frac{\partial p}{\partial x_{i}}\right) \mathrm{d} V=\int_{V} \frac{\partial \mathbf{N}_{p}}{\partial x_{i}}\left(\boldsymbol{\Pi}_{i}-\tau_{0} \frac{\partial p}{\partial x_{i}}\right) \mathrm{d} V \\
& -\oint \mathbf{N}_{p}\left(\boldsymbol{\Pi}_{i}-\tau_{0} \frac{\partial p}{\partial x_{i}}\right) v_{i} \mathrm{~d} \Gamma
\end{aligned}
$$

The boundary terms of Equation (60) are normally neglected [22], remaining:

$$
\begin{aligned}
\mathbf{S P} & =\int_{V} \frac{\partial \mathbf{N}_{p}}{\partial x_{i}}\left(\boldsymbol{\Pi}_{i}-\tau_{0} \frac{\partial p}{\partial x_{i}}\right) \mathrm{d} V \\
& =\left(\int_{V} \frac{\partial \mathbf{N}_{p}}{\partial x_{i}} \mathbf{N} \mathrm{d} V \boldsymbol{\Pi}-\int_{V} \frac{\partial \mathbf{N}_{p}}{\partial x_{i}} \tau_{0} \frac{\partial \mathbf{N}_{p}^{\mathrm{T}}}{\partial x_{i}} \mathrm{~d} V \mathbf{P}\right)=\left(\mathbf{D}^{\mathrm{T}} \mathbf{M}_{\tau_{0}}^{-1} \mathbf{D}-\mathbf{L}_{\tau_{0}}\right) \mathbf{P}
\end{aligned}
$$


or simply

$$
\mathbf{S}=\mathbf{D}^{\mathrm{T}} \mathbf{M}_{\tau_{0}}^{-1} \mathbf{D}-\mathbf{L}_{\tau_{0}}
$$

Note that $\mathbf{D}$ is different from $\mathbf{B}$ and also from $\mathbf{B}^{\mathrm{T}}$.

Introducing matrix $\mathbf{S}$ into Equations (44)-(46) reads:

$$
\begin{aligned}
\left(\frac{\mathbf{M}_{\rho}}{\Delta t}+\mathbf{K}\right) \tilde{\mathbf{V}} & =\left(\mathbf{F}+\frac{\mathbf{M}_{\rho}}{\Delta t} \mathbf{V}^{n}+\mathbf{B} \mathbf{P}_{0}\right) \\
\left(-\mathbf{L}_{\tau}+\mathbf{D}^{\mathrm{T}} \mathbf{M}_{\tau_{0}}^{-1} \mathbf{D}-\mathbf{L}_{\tau_{0}}\right) \mathbf{P} & =\mathbf{B}^{\mathrm{T}} \tilde{\mathbf{V}}-\mathbf{L}_{\tau} \mathbf{P}_{0} \\
\left(\frac{\mathbf{M}_{\rho}}{\Delta t}+\mathbf{K}\right)(\mathbf{V}-\tilde{\mathbf{V}}) & =\mathbf{B} \delta \mathbf{P}
\end{aligned}
$$

The stabilization terms $\left(\mathbf{D}^{\mathrm{T}} \mathbf{M}_{\tau_{0}}^{-1} \mathbf{D}-\mathbf{L}_{\tau_{0}}\right) \mathbf{P}$ may be solved explicitly for the term $\left(\mathbf{D}^{\mathrm{T}} \mathbf{M}_{\tau_{0}}^{-1} \mathbf{D}\right) \mathbf{P}$ using the previous iteration of the pressure vector $\mathbf{P}^{k-1}$. In other words:

$$
\left(\mathbf{D}^{\mathrm{T}} \mathbf{M}_{\tau_{0}}^{-1} \mathbf{D}-\mathbf{L}_{\tau_{0}}\right) \mathbf{P} \approx \mathbf{D}^{\mathrm{T}} \mathbf{M}_{\tau_{0}}^{-1} \mathbf{D} \mathbf{P}^{k-1}-\mathbf{L}_{\tau_{0}} \mathbf{P}
$$

The four steps stabilized algorithm remains:

$$
\begin{array}{|lc|}
\text { I) } & \left(\frac{\mathbf{M}_{\rho}}{\Delta t}+\mathbf{K}\right) \tilde{\mathbf{V}}=\left(\mathbf{F}+\frac{\mathbf{M}_{\rho}}{\Delta t} \mathbf{V}^{n}+\mathbf{B} \mathbf{P}_{0}\right) \\
\text { II) } & \mathbf{M}_{\tau_{0}} \boldsymbol{\Pi}^{k-1}=\mathbf{D} \mathbf{P}^{k-1} \\
\text { III }) & \left(-\mathbf{L}_{\tau}-\mathbf{L}_{\tau_{0}}\right) \mathbf{P}=\mathbf{B}^{\mathrm{T}} \tilde{\mathbf{V}}-\mathbf{L}_{\tau} \mathbf{P}_{0}-\mathbf{D}^{\mathrm{T}} \mathbf{\Pi}^{k-1} \\
\text { IV) } & \left(\frac{\mathbf{M}_{\rho}}{\Delta t}+\mathbf{K}\right)(\mathbf{V}-\tilde{\mathbf{V}})=\mathbf{B} \boldsymbol{\delta} \mathbf{P}
\end{array} \overrightarrow{\mathbf{V}}
$$

Equations (67)-(70) may be solved implicitly, which requires the solution of four systems of equations: two with the same matrix $\left(\mathbf{M}_{\rho} / \Delta t\right)+\mathbf{K}$ (which may be triangularized once), one with matrix $\mathbf{M}_{\tau_{0}}$ and the fourth equation is a Laplace system for the pressure involving matrix $\mathbf{L}_{\tau}+\mathbf{L}_{\tau_{0}}$.

Equation (68) is normally solved explicitly with a lumped form of matrix $\mathbf{M}_{\tau_{0}}$.

Several strategies may be used to solve Equations (67)-(70) as a non-linear problem. One is to take $\mathbf{P}_{0}=\mathbf{P}^{k-1}$ in Equation (67). In this case, the method is called predictor-corrector and the results converge to the monolithic solution. Other possibility is to take $\mathbf{P}^{k-1}=\mathbf{P}_{0}$ in Equation (68). In this case, the static condensation error introduced in Equation (43) does not disappear, introducing an additional stabilization term. The convergence is fast but the result is not equal to the monolithic one. 
Considering the $\mathbf{K}$ matrix negligible in front of $\mathbf{M}_{\rho} / \Delta t$ (which is always true for small time steps) Equation (70) may be also solved explicitly with a lumped mass matrix $\mathbf{M}_{D}$.

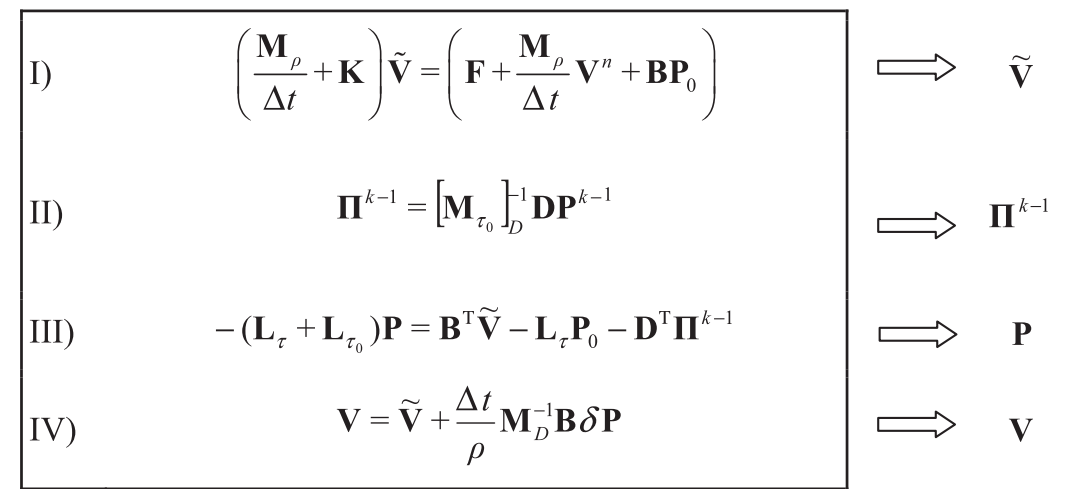

It must be noted that in both cases, Equation (69) as well as Equation (73) have a Laplace form. Then in order to be solved, essential boundary conditions must be introduced in the pressure term in order to avoid the singularity of the Laplace matrix. The standard approach is to impose the pressure on the free-surface boundaries or to impose an arbitrary pressure value in one node in the case of closed domains.

Observing Equations (69) and (73) two different parameters multiply the Laplace matrix L. One of them is $\tau_{0}$, the stabilization parameter. The other one is $\tau$. This one is not a stabilization parameter. It is a parameter that comes from the static condensation of Equation (43). This parameter is multiplying a Laplace matrix by $\boldsymbol{\delta P}$. This means that when the convergence is achieved the $\tau$ value does not affect the results. This is not the case for $\tau_{0}$, which is multiplying the difference between the pressure gradient and its projection. This difference is not necessarily zero after convergence, which means that $\tau_{0}$ stabilizes the final results.

Nevertheless, the terms introduced while $\mathbf{L}_{\tau} \boldsymbol{\delta} \mathbf{P}$ is different than zero are important to achieve convergence in FSI problems with added-mass effects. Although both parameters are equal $\left(\tau=\tau_{0}\right)$ they are expressed separately in the Laplace equation in order to explain the different meaning of each of them.

\section{EVALUATION OF THE LAPLACE MATRIX L $(\tau)$ FOR FSI PROBLEMS}

When solving an incompressible fluid-elastic solid interaction problem, the incompressible condition (2) is only applied to the fluid domains. This means that the discretized form $\mathbf{B}_{i}^{\mathrm{T}} \mathbf{V}_{i}=0$ only affects some DOF.

Let us call $n_{P}$ the total DOF corresponding to the pressure, $n_{V}$ the total DOF corresponding to the velocity, $n_{\mathrm{s}}$ the velocity DOF corresponding to the solid exclusively (without the interfaces), $n_{\mathrm{f}}$ the velocity DOF corresponding exclusively to the fluid (without the interfaces), and $n_{\text {sf }}$ the velocity DOF of the interfaces solid-fluid. For simplicity, we will consider only conforming meshes, this means that the interface between the fluid and the solid has exactly the same number of nodes.

Then matrix $\mathbf{B}^{\mathrm{T}}$ is a matrix of $n_{P}$ files and $n_{V}$ columns, but all the columns corresponding to the $n_{\mathrm{s}}$ solid DOF are zero. Matrix $\mathbf{B}^{\mathrm{T}}$ has non-zero columns in the DOF corresponding to the fluid domain and the interfaces. 
On the other hand, matrix $\mathbf{M}_{T}^{-1}$ is a diagonal matrix, with terms:

$$
\mathbf{M}_{T}^{-1}=\left[\left(\frac{\rho_{\mathrm{f}}}{\Delta t}+\frac{\mu}{h^{2}}+\frac{\left|\mathbf{V}^{n+1}-\mathbf{V}_{m}\right|}{h}\right) \mathbf{M}_{D}\right]^{-1}
$$

in the $n_{\mathrm{f}} \mathrm{DOF}$

$$
\mathbf{M}_{T}^{-1}=\left[\left(\frac{\rho_{\mathrm{s}}}{\Delta t}+\frac{G \Delta t}{J h^{2}}+\frac{\lambda \Delta t}{J h^{2}}\right) \mathbf{M}_{D}\right]^{-1}
$$

in the $n_{\mathrm{S}} \mathrm{DOF}$ and

$$
\mathbf{M}_{T}^{-1}=\left[\left(\frac{\rho_{\mathrm{f}}}{\Delta t}+\frac{\mu}{h^{2}}+\frac{\left|\mathbf{V}^{n+1}-\mathbf{V}_{m}\right|}{h}+\frac{\rho_{\mathrm{s}}}{\Delta t}+\frac{G \Delta t}{J h^{2}}+\frac{\lambda \Delta t}{J h^{2}}\right) \mathbf{M}_{D}\right]^{-1}
$$

in the $n_{\text {sf }}$ DOF.

Performing the double product $\mathbf{B}^{\mathrm{T}} \mathbf{M}_{T}^{-1} \mathbf{B}$, all the terms corresponding to the $n_{\mathrm{s}}$ DOF are zero. Matrix $\mathbf{L}_{\tau}$ may be written as:

$$
\mathbf{L}_{\tau}=\mathbf{B}^{\mathrm{T}} \mathbf{M}_{T}^{-1} \mathbf{B} \approx \mathbf{L}_{\tau_{\mathrm{f}}}+\overline{\mathbf{L}_{\tau_{\mathrm{s}}}}
$$

Where $\mathbf{L}_{\tau_{\mathrm{f}}}$ is the standard Laplace matrix corresponding to the fluid domains including the interfaces (see Equations (35), (36) and (43)):

$$
\mathbf{L}_{\tau_{\mathrm{f}}}=\sum\left(\tau_{\mathrm{f}}^{e} \mathbf{L}^{e}\right)
$$

defined only on the fluid domain and $\overline{\mathbf{L}_{\tau_{\mathrm{s}}}}$ is a Laplace matrix corresponding only to the fluid-solid interface

$$
\overline{\mathbf{L}_{\tau_{\mathrm{s}}}}=\sum\left(\tau_{\mathrm{s}} \overline{\mathbf{L}}^{e}\right)
$$

where

$$
\overline{\mathbf{L}}^{e}=\left[\int_{V} \frac{\partial \mathbf{N}_{\mathrm{sf}}}{\partial x_{j}} \frac{\partial \mathbf{N}_{\mathrm{sf}}^{\mathrm{T}}}{\partial x_{j}} \mathrm{~d} V\right]_{\mathrm{s}}
$$

is the Laplace matrix of the solid elements evaluated only with the shape functions $\mathbf{N}_{\mathrm{sf}}$ that are different from zero on the fluid-solid interface.

Equation (78) may be also written as:

$$
\mathbf{L}_{\tau}=\mathbf{L}_{\tau_{\mathrm{f}}}+\beta \overline{\mathbf{L}_{\tau_{\mathrm{f}}}}
$$

with

$$
\beta=\frac{\tau_{\mathrm{s}}}{\tau_{\mathrm{f}}}=\frac{\frac{\rho_{\mathrm{f}}}{\Delta t}+\frac{\mu}{h^{2}}+\frac{\left|\mathbf{V}^{n+1}-\mathbf{V}_{m}\right|}{h}}{\frac{\rho_{\mathrm{s}}}{\Delta t}+\frac{G \Delta t}{J h^{2}}+\frac{\lambda \Delta t}{J h^{2}}}
$$

and

$$
\overline{\mathbf{L}_{\tau_{\mathrm{f}}}}=\sum\left(\tau_{\mathrm{f}} \overline{\mathbf{L}}^{e}\right)
$$


This means that the Laplace interface matrix $\overline{\mathbf{L}_{\tau_{\mathrm{f}}}}$ may be neglected for small values of the $\beta$ parameter. This is, for instance, the case when $\rho_{\mathrm{s}} \gg \rho_{\mathrm{f}}$ and the added-mass effect is not present. However, for other physical properties, the $\beta$ parameter may not be negligible and the Laplace interface matrix must be evaluated in order to obtain good results.

For instance, for non-viscous flows $(\mu \approx 0)$, a Lagrangian formulation $\left(\mathbf{V} \approx \mathbf{V}_{m}\right)$ and small displacements $(J \approx 1)$, the $\beta$ parameter is:

$$
\beta=\frac{\rho_{\mathrm{f}}}{\left(\rho_{\mathrm{s}}+\frac{(G+\lambda) \Delta t^{2}}{h^{2}}\right)}
$$

This means that $\beta \rightarrow 0$ for large values of $\frac{(G+\lambda) \Delta t^{2}}{h^{2}}$, that is for the large $\Delta t$, the large shear stiffness $G$ and $\lambda$ and for small mesh size $h$. However, $\beta$ is not negligible for $\rho_{\mathrm{f}} / \rho_{\mathrm{s}} \approx 1$ or if we are using small time steps or the solid material is very soft.

In the examples we will show that the $\overline{\mathbf{L}_{\tau_{\mathrm{f}}}}$ matrix improves the convergence in the following cases:

(1) $\rho_{\mathrm{f}} \geqslant \rho_{\mathrm{s}}$ (classical added-mass effect).

(2) Small $\Delta t$ (this means that instabilities do not disappear by decreasing the time step).

(3) Soft materials (i.e. bio-medical materials).

Equation (83) shows that the evaluation of $\beta$ is rather more complicated than a simple ratio between the fluid and the solid densities. In general, $\beta$ has small values, but its inclusion improves considerable the convergence rate in all cases. Also in the cases where the added-mass effect is strong its inclusion is crucial to achieve convergence.

\section{THE PARTITIONED (OR STAGGERED) SCHEME}

Partitioned schemes are based on dividing the original FSI problem into two parts: the solid one and the fluid one. The division is performed independently of using or not sub-iterations in a strongly coupled partitioned scheme or in a loosely coupled one. The idea is exactly the same as for the pressure segregation described above for which the system was split in the velocity and pressure unknowns. Now the same system of equations is divided into the solid unknowns (for instance the velocity or the displacements) and the fluid unknowns (normally the velocity and the pressure). Both systems are solved separately. Some recent work in this topic may be found in $[12,15,25]$.

Let us call $\mathbf{V}_{\mathrm{s}}$ the vector containing the solid unknowns, $\mathbf{V}_{\mathrm{f}}$ and $\mathbf{P}$ the vectors containing the fluid unknowns, not including the common solid-fluid unknowns, and $\mathbf{V}_{\text {sf }}$ the vector including the common solid-fluid unknowns.

The transfer of information occurs on the boundary $\Gamma_{S F}$ by using techniques that guarantee momentum and energy conservation [2]. For staggered algorithms the use of non-matching meshes is a common practice since both systems, fluid and structure, are completely decoupled. In this work only conformal (matching) meshes will be analysed. However, we consider that the main result concerning the convergence properties of the interface Laplace matrix is also valid for non-matching meshes. 
The classical boundary conditions at the interface are:

$$
\begin{aligned}
\left(\mathbf{V}_{\mathrm{f}}-\mathbf{V}_{\mathrm{s}}\right)^{\mathrm{T}}=0 & \text { on } \Gamma_{\mathrm{f}-\mathrm{s}} \\
\boldsymbol{\sigma}_{\mathrm{f}}+\mathbf{t}_{\mathrm{s}}=0 & \text { on } \Gamma_{\mathrm{f}-\mathrm{s}}
\end{aligned}
$$

where Equation (86) represents the consistency condition. Since the interface is modelled using a fully Lagrangian frame of reference, this condition guarantees that the fluid and solid meshes will remain tightly coupled along the FSI interface. Equation (87) represents the equilibrium of stresses along the interfaces.

The original FSI equation (15) may be then written as:

$$
\left[\begin{array}{cccc}
\left(\frac{\mathbf{M}_{\rho}}{\Delta t}+\mathbf{K}\right)_{\mathrm{s}} & \left(\frac{\mathbf{M}_{\rho}}{\Delta t}+\mathbf{K}\right)_{\mathrm{sf}} & 0 & 0 \\
\left(\frac{\mathbf{M}_{\rho}}{\Delta t}+\mathbf{K}\right)_{\mathrm{sf}}^{\mathrm{T}} & \left(\frac{\mathbf{M}_{\rho}}{\Delta t}+\mathbf{K}\right)_{\mathrm{ssf}} & \left(\frac{\mathbf{M}_{\rho}}{\Delta t}+\mathbf{K}\right)_{\mathrm{fs}} & -\mathbf{B}_{\mathrm{sf}} \\
0 & \left(\frac{\mathbf{M}_{\rho}}{\Delta t}+\mathbf{K}\right)_{\mathrm{fs}}^{\mathrm{T}} & \left(\frac{\mathbf{M}_{\rho}}{\Delta t}+\mathbf{K}\right)_{\mathrm{f}} & -\mathbf{B}_{\mathrm{f}} \\
0 & -\mathbf{B}_{\mathrm{sf}}^{\mathrm{T}} & -\mathbf{B}_{\mathrm{f}}^{\mathrm{T}} & 0
\end{array}\right]\left[\begin{array}{c}
\mathbf{V}_{\mathrm{s}} \\
\mathbf{V}_{\mathrm{sf}} \\
\mathbf{V}_{\mathrm{f}} \\
\mathbf{P}
\end{array}\right]=\left[\begin{array}{c}
\left(\mathbf{F}+\frac{\mathbf{M}_{\rho}}{\Delta t} \mathbf{V}^{n}\right)_{\mathrm{s}} \\
\left(\mathbf{F}+\frac{\mathbf{M}_{\rho}}{\Delta t} \mathbf{V}^{n}\right)_{\mathrm{sf}} \\
\left(\mathbf{F}+\frac{\mathbf{M}_{\rho}}{\Delta t} \mathbf{V}^{n}\right)_{\mathrm{f}} \\
0
\end{array}\right]
$$

In the monolithic method with pressure segregation previously described, Equation (88) was partitioned into two parts: the three first rows and columns and in the fourth row and column. In classical staggered methods, Equation (88) is partitioned also into two parts the first two rows and columns and the third and fourth rows and columns.

For each sub-iteration, the static condensation of the terms

$$
\mathbf{B}_{\text {sf }}^{\mathrm{T}}\left(\frac{\mathbf{M}}{\Delta t}+\mathbf{K}\right)_{\text {ssff }}^{-1} \mathbf{B}_{\text {sf }}
$$

must be taken into account when the added-mass effect is strong. Remember from Section 3 that this term is placed on the left-hand side of the pressure equation (Equation (45)). In the partitioned scheme the classical Neumann boundary conditions are imposed on $\Gamma_{\mathrm{f}-\mathrm{s}}$ and thus Equation (89) is still of great importance.

Using the same conclusion reached in Section 4, an interface Laplace matrix $\beta \overline{\mathbf{L}_{\tau_{\mathrm{f}}}}$ must be added when solving the incompressible part of the domain. Since the meshes on the interface may be non-matching special care has to be taken when Equations (75)-(77) are evaluated. In the general case Equation (77) takes the form:

$$
\mathbf{M}_{T}^{-1}=\left[\left(\frac{\rho_{\mathrm{f}}}{\Delta t}+\frac{\mu}{h^{2}}+\frac{\left|\mathbf{V}^{n+1}-\mathbf{V}_{m}\right|}{h}\right) \mathbf{M}_{D \mathrm{f}}+\left(\frac{\rho_{\mathrm{s}}}{\Delta t}+\frac{G \Delta t}{J h^{2}}+\frac{\lambda \Delta t}{J h^{2}}\right) \mathbf{M}_{D \mathrm{~s}}\right]^{-1}
$$

In conclusion matrix $\beta \overline{\mathbf{L}_{\tau_{\mathrm{f}}}}$ must be added to the fluid equation independently of the method used to solve the incompressibility condition. This means that independently of using or not pressure segregation, the fluid solution must include the interface Laplace matrix. This is because, when using a partitioned solution, pressure segregation is implicitly included in the procedure as explained in Equation (88). 
Partitioned algorithms are in fact more complicated to approximate with a Laplace matrix than monolithic ones using pressure segregation due to the fact that there are other connecting matrices in addition to $\mathbf{B}_{\mathrm{sf}}$. For example, the matrix corresponding to the velocity inside the fluid and the interfaces after static condensation, this matrix yields the terms:

$$
\left(\frac{\mathbf{M}}{\Delta t}+\mathbf{K}\right)_{\mathrm{sf}}^{\mathrm{T}}\left(\frac{\mathbf{M}}{\Delta t}+\mathbf{K}\right)_{\mathrm{ssff}}^{-1}\left(\frac{\mathbf{M}}{\Delta t}+\mathbf{K}\right)_{\mathrm{sf}}
$$

Fortunately, these matrices are negligible in front of the pressure forces on the interfaces and therefore are normally neglected.

To satisfy the boundary conditions (86), (87) and therefore guarantee energy conservation through the interface, the staggered approach uses sub-iterations where $\mathbf{P}, \mathbf{V}_{\mathrm{f}}, \mathbf{V}_{\mathrm{s}}$ are updated at each substep. This procedure is essential when added-mass effects are present providing a stronger coupling between the fluid and the solid variables. For the case of aero-elasticity, an explicit coupling via a single sub-step may provide enough stability. Splitting Equation (88) for the staggered approach results in:

$$
\begin{aligned}
\left(\frac{\mathbf{M}_{\rho}}{\Delta t}+\mathbf{K}\right)_{\mathrm{s}} \mathbf{V}_{\mathrm{s}} & =\left(\mathbf{F}_{\left(P_{\mathrm{sf}}\right)}+\frac{\mathbf{M}_{\rho}}{\Delta t} \mathbf{V}^{n}\right)_{\mathrm{s}} \\
\left(\frac{\mathbf{M}_{\rho}}{\Delta t}+\mathbf{K}\right)_{\mathrm{f}} \mathbf{V}_{\mathrm{f}}-\mathbf{B}_{\mathrm{f}} \mathbf{P}_{\left(V_{\mathrm{sf}}\right)} & =\left(\mathbf{F}+\frac{\mathbf{M}_{\rho}}{\Delta t} \mathbf{V}^{n}\right)_{\mathrm{f}} \\
\mathbf{B}_{\mathrm{sf}}^{\mathrm{T}} \mathbf{V}_{\mathrm{sf}}+\mathbf{B}_{\mathrm{f}}^{\mathrm{T}} \mathbf{V}_{\mathrm{f}} & =0
\end{aligned}
$$

As mentioned above, pressure segregation for the staggered approach implies a one-step solution for the solid problem and a fractional step method for the fluid variables.

Following a similar procedure to the one described in Section 3, Equations (92)-(94) become:

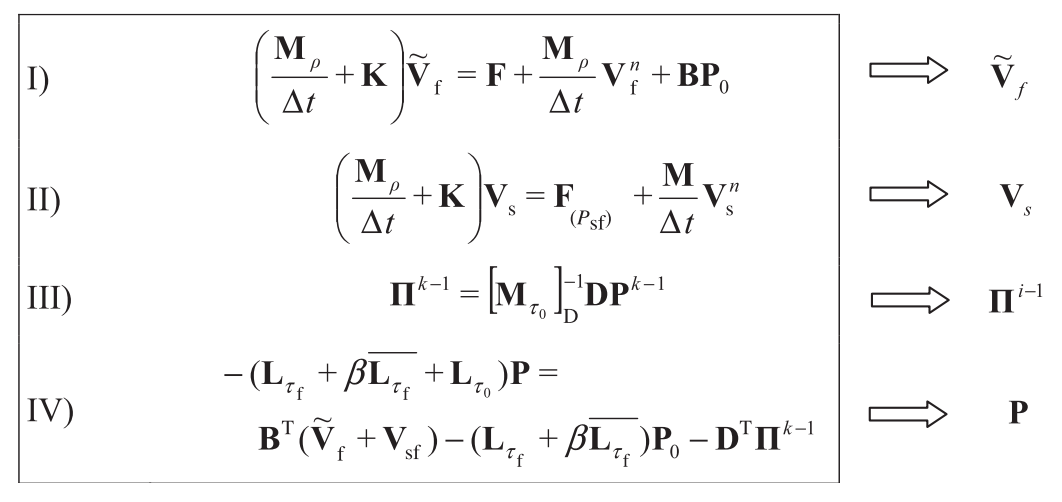

To simplify the notation the lower index for the matrices has been removed implying that the matrices for the fluid problem are computed on the fluid domain and similarly for the solid.

In the examples presented in the next section, Equations (95)-(98) are solved with: $\mathbf{P}^{k-1}=\mathbf{P}_{0}$ in Equation (97) and $\mathbf{P}_{0}=\mathbf{P}_{n}$ in Equation (95) at each time step. Then, steps I and III are performed only once per time step. Sub-stepping iterations are performed between steps III and IV (Equations (97) and (98)) and the solid solver. Convergence is achieved when $\left|\mathbf{V}_{\mathrm{sf}}^{k}-\mathbf{V}_{\mathrm{sf}}^{k-1}\right|_{p}<\varepsilon$. 
The iterations performed between the steps II and IV involve a simple fixed-point method. Thus, no acceleration technique has been used to improve the convergence of the method.

The time integration on the solid will not be considered in the present analysis. For instance, the Newmark method may be applied in this case. It is important to notice that different $\Delta t$ could be used in the fluid and solid domains. If this is the case the time step has to be synchronized between both solvers.

Matrix $\beta \overline{\mathbf{L}_{\tau_{f}}}$ has to be computed in the same way as for the monolithic approach. For nonmatching meshes some considerations have to be taken into account when dealing with the matrices on the interface elements. These considerations will not be addressed here. A simplified way to deal with this situation is to evaluate the interface matrix

$$
\beta \overline{\mathbf{L}_{\tau_{\mathrm{f}}}}=\beta \tau_{\mathrm{f}} \int_{V} \frac{\partial \mathbf{N}_{\mathrm{sf}}}{\partial x_{j}} \frac{\partial \mathbf{N}_{\mathrm{sf}}^{\mathrm{T}}}{\partial x_{j}} \mathrm{~d} V
$$

which must be computed with the shape functions of the solid and fluid domain using only the shape functions of the fluid domain:

$$
\beta \overline{\mathbf{L}_{\tau_{\mathrm{f}}}}=\beta \tau_{\mathrm{f}}\left[\int_{V} \frac{\partial \mathbf{N}_{\mathrm{sf}}}{\partial x_{j}} \frac{\partial \mathbf{N}_{\mathrm{sf}}^{\mathrm{T}}}{\partial x_{j}} \mathrm{~d} V\right]_{\mathrm{fs}} \approx \beta \tau_{\mathrm{f}}\left[\int_{V} \frac{\partial \mathbf{N}_{\mathrm{f}}}{\partial x_{j}} \frac{\partial \mathbf{N}_{\mathrm{f}}^{\mathrm{T}}}{\partial x_{j}} \mathrm{~d} V\right]_{\mathrm{f}}
$$

This approach is valid when both meshes are sufficiently similar. All the validation examples approximated with the staggered approach use Equation (99) since the fluid and solid nodes almost match at the interface to avoid introducing errors from this approximation.

\section{NUMERICAL EXAMPLES}

\subsection{Fluid column interacting with an elastic solid bottom}

The first example is a very simple 1D problem for which an analytical result can be easily obtained. Nevertheless from the numerical point of view it has some convergence problems. The example is ideal to test different materials and time step sizes in order to check the validity of the algorithm proposed, in particular the effectiveness of the interface matrix to improve the convergence rate.

The example consists in an incompressible water column over an elastic solid (Figure 2).

Both column walls have the horizontal displacement constrained (plane strain). The upper line is a free surface and the bottom one has the displacement constrained. Initially, the example had the following physical properties: $\rho_{\mathrm{s}}=1500\left(\mathrm{~kg} / \mathrm{m}^{3}\right) ; \rho_{\mathrm{f}}=1000\left(\mathrm{~kg} / \mathrm{m}^{3}\right) ; E_{\mathrm{s}}=2.3 \times 10^{5}\left(\mathrm{~kg} / \mathrm{m} \mathrm{s}^{2}\right)$; $v=0.4$ and $\mu_{\mathrm{f}}=0$. The gravity was fixed to $g=-10\left(\mathrm{~m} / \mathrm{s}^{2}\right)$ in the vertical direction and the geometry was discretized as a $2 \mathrm{D}$ problem using a mesh of $4 \times 2003$-node linear triangles with $h=0.025(\mathrm{~m})$.

Note that a Young modulus of order $E_{\mathrm{s}}=2.3 \times 10^{5}\left(\mathrm{~kg} / \mathrm{m} \mathrm{s}^{2}\right)$ corresponds to a very soft material, probably a muscle-like heart material. For instance, for rubber $E_{\text {rubber }} \cong 7 \times 10^{6}\left(\mathrm{~kg} / \mathrm{m} \mathrm{s}^{2}\right)$; a human cartilage $E_{\text {cartiage }} \cong 2 \times 10^{7}\left(\mathrm{~kg} / \mathrm{m} \mathrm{s}^{2}\right)$ and for a human bone $E_{\text {cartiage }} \cong 2 \times 10^{9}\left(\mathrm{~kg} / \mathrm{m} \mathrm{s}^{2}\right)$. For steel $E_{\text {steel }} \cong 2 \times 10^{11}\left(\mathrm{~kg} / \mathrm{m} \mathrm{s}^{2}\right)$ and for a very stiff material as sapphire $E_{\text {sapphire }} \cong 4.2 \times 10^{11}\left(\mathrm{~kg} / \mathrm{m} \mathrm{s}^{2}\right)$.

Figure 3(a) shows the vertical displacements in $(\mathrm{cm})$ of any fluid particle as a function of time. Figure 3(b) shows the same vertical displacements of the entire domain at different time steps. The perfect vertical line corresponds to the incompressible bar. This very simple result is not easy 


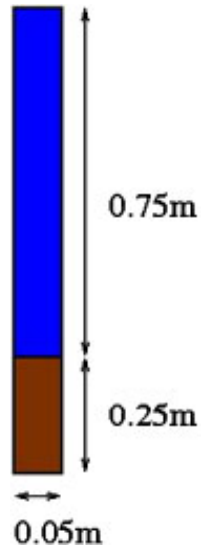

Figure 2. Water column over an elastic beam.

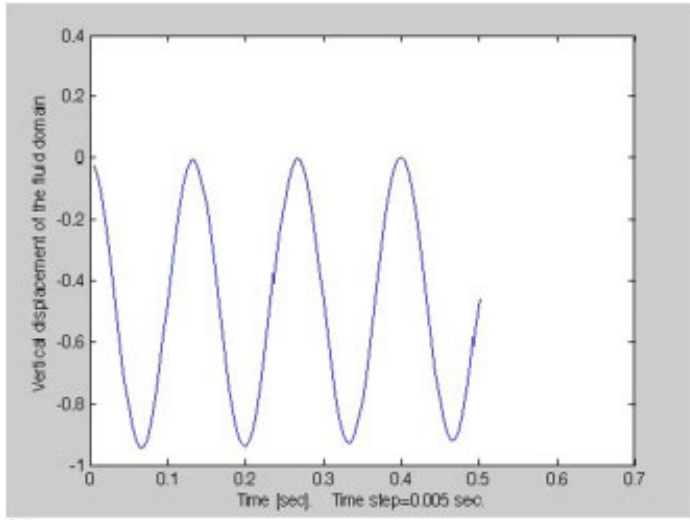

(a)

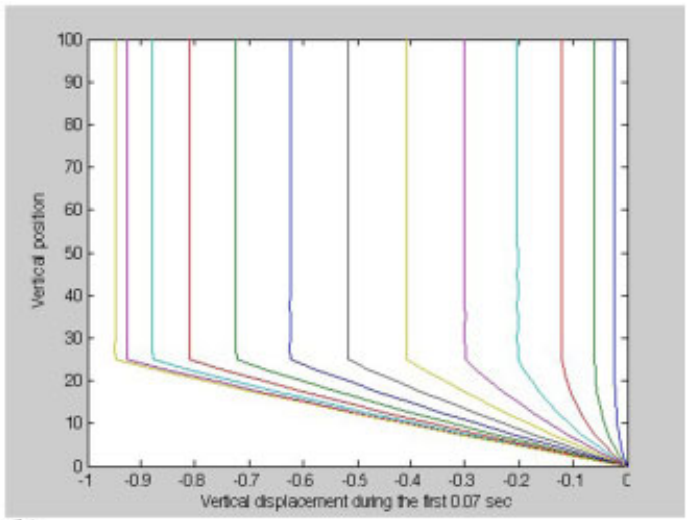

(b)

Figure 3. Water column over an elastic beam: (a) vertical displacement versus time at any fluid point and (b) vertical displacement in the total domain for different time steps.

to obtain. For instance, the fractional-step method $\left(\mathbf{P}_{0}=\left.\mathbf{P}_{0}\right|_{t=t^{n}}\right.$ in Equation (71)) with exactly the same time step and the same convergence rate gives the results presented in Figure 4. The lack of convergence to the monolithic scheme is the reason of these oscillations.

The numerical solution does not converge when the interface Laplace matrix $\beta \overline{\mathbf{L}_{\tau_{\mathrm{f}}}}$ is neglected $(\beta=0)$. The best way to see the importance of this matrix is to study different situations with different densities, different Young modulae and different time step sizes.

Table I shows the performance of the algorithm for a stiff material with a Young modulus similar to steel and different density ratio. We can observe that $\beta=0$ is acceptable only for density rates larger than 6 . The number of iteration to achieve the same error is equal to 20 in all cases for $\beta \neq 0$ but it is larger than 40 iterations for $\beta=0$. This means that even in the case of a FSI of steel and water $\left(\left(\rho_{\mathrm{s}} / \rho_{\mathrm{f}}\right)=7, \beta \neq 0\right)$ must be used. Probably only in aero-elasticity applications where the density rate is larger than 1000 the omission of the interface Laplace matrix is justified. 


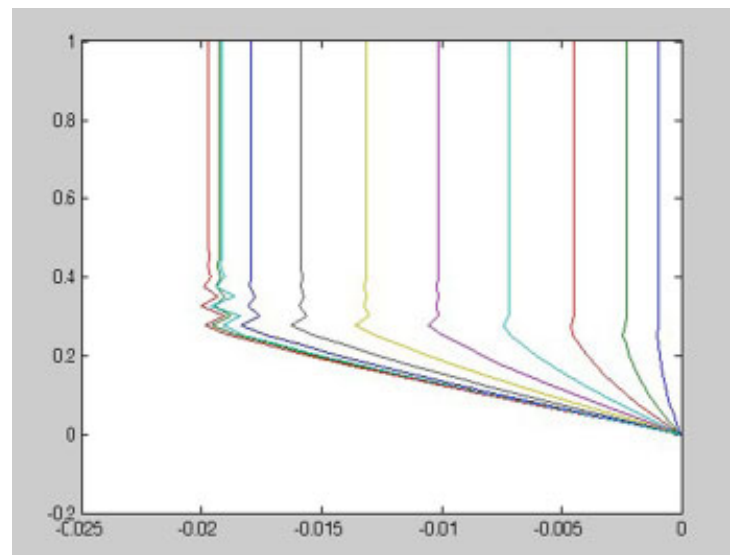

Figure 4. Water column over an elastic beam: vertical displacement in the total domain at different time steps for the Fractional Step Method.

Table I. Iterations to achieve convergence for different density ratio.

\begin{tabular}{lll}
\hline$\frac{\rho_{\mathrm{s}}}{\rho_{\mathrm{f}}}$ & \multicolumn{1}{c}{$\beta \neq 0$} & \multicolumn{1}{c}{$\beta=0$} \\
\hline 10 & 20 iterations & 20 iterations \\
7 & 20 iterations & more than 40 iterations \\
6 & 20 iterations & more than 40 iterations \\
5 & 20 iterations & does not converge \\
3 & 19 iterations & does not converge \\
1 & 18 iterations & does not converge \\
\hline
\end{tabular}

$E_{S}=2 \times 10^{11}\left(\mathrm{~kg} / \mathrm{m} \mathrm{s}^{2}\right) ; v=0.3 \Delta t=10^{-5}(\mathrm{~s})$.

Table II shows the same problem with different stiffness properties for the elastic domain but with the density rate fixed to one. This means equal density in the elastic solid as in the fluid. Owing to the oscillatory behaviour of the problem, the time step $(\Delta t(\mathrm{~s}))$ must be changed in order to achieve reasonable time integration with a minimum of time steps in each oscillation. We can observe that only for very high Young modulus the case with $\beta=0$ converge. There is an exception for $E=2 \times 10^{6}$ and $\Delta t=1 \times 10^{-2}$. Using $\beta=0$ the algorithm converges in 33 iterations. We must say, however, that in order to obtain an acceptable result from the time integration point of view the correct time step for this case is less than $1 \times 10^{-2}$. Nevertheless, the same stiffness with $\Delta t=1 \times 10^{-3}$ does not converge for $\beta=0$. This is consistent with the well-known conclusion that for equal density rates, the added-mass effect is so important in all cases that without the interface Laplace matrix or any other artifice, the problem does not converge.

However, the most worrisome results are those presented in Table III. They correspond to a standard steel elastic modulus with a density rate equal to 7 . We use first the correct time step size for this kind of problem and we see that both formulations for $\beta=0$ and $\beta \neq 0$ converge reasonably well in 21 iterations. Nevertheless, halving the time step, the number of iterations with $\beta=0$ duplicates. Decreasing the time step more and more the method with $\beta=0$ does not converge, 
Table II. Iterations to achieve convergence for different Young modulus.

\begin{tabular}{lll}
\hline$E\left(\mathrm{~kg} / \mathrm{m} \mathrm{s}^{2}\right) ;(\Delta t(\mathrm{~s}))$ & \multicolumn{1}{c}{$\beta \neq 0$} & \multicolumn{1}{c}{$\beta=0$} \\
\hline $2 \times 10^{13} ;\left(0.2 \times 10^{-5}\right)$ & 10 iterations & more than 40 iterations \\
$2 \times 10^{12} ;\left(0.5 \times 10^{-5}\right)$ & 14 iterations & does not converge \\
$2 \times 10^{11} ;\left(1 \times 10^{-5}\right)$ & 18 iterations & does not converge \\
$2 \times 10^{8} ;\left(1 \times 10^{-4}\right)$ & 40 iterations & does not converge \\
$2 \times 10^{7} ;\left(1 \times 10^{-3}\right)$ & 36 iterations & does not converge \\
$2 \times 10^{6} ;\left(1 \times 10^{-3}\right)$ & 40 iterations & does not converge \\
$2 \times 10^{6} ;\left(1 \times 10^{-2}\right)$ & 34 iterations & 33 iterations \\
$2 \times 10^{5} ;\left(1 \times 10^{-2}\right)$ & 36 iterations & does not converge \\
\hline$\rho_{\mathrm{s}} / \rho_{\mathrm{f}}=1 ; v=0.3$. &
\end{tabular}

Table III. Iterations to achieve convergence for different time steps.

\begin{tabular}{lll}
\hline$\Delta t(\mathrm{~s})$ & \multicolumn{1}{c}{$\beta \neq 0$} & \multicolumn{1}{c}{$\beta=0$} \\
\hline $2 \times 10^{-5}$ & 23 iterations & 21 iterations \\
$1 \times 10^{-5}$ & 20 iterations & more than 40 iterations \\
$0.75 \times 10^{-5}$ & 18 iterations & does not converge \\
$0.5 \times 10^{-5}$ & 16 iterations & does not converge \\
$0.25 \times 10^{-5}$ & 11 iterations & does not converge \\
\hline$\rho_{\mathrm{s}} / \rho_{\mathrm{f}}=7 ; E_{S}=2 \times 10^{11}\left(\mathrm{~kg} / \mathrm{m} \mathrm{s}^{2}\right) v=0.3$. &
\end{tabular}

while the algorithm with the interface Laplace matrix converges with a decreasing number of iterations as expected.

This example shows that even when classical materials like steel and water are involved, the use of the interface Laplace matrix is recommended to avoid possible difficulties when the time step is smaller than necessary.

The problems were tested with both methods: monolithic with pressure segregation and with a strongly coupled partitioned scheme with similar conclusions in both cases.

\subsection{Mesh sensitivity analysis}

The objective of this example is to show the influence of the FSI algorithm presented in this work for different mesh configurations. In particular, we are interested in studying the convergence rate of the coupled problem for the case of a partitioned approach and the effect of the $\beta$ parameter for different mesh sizes.

When solving FSI problems with large deformations using a PFEM-ALE formulation [26, 27], the mesh moving algorithm will eventually distort the mesh in such a way that the element quality becomes unacceptable. In some occasions, specially in the presence of rotations, the mesh moving algorithm cannot deal with the extreme deformations that will finally result in elements inversion and negative Jacobians. For this reason a robust non-linear FSI solver should provide a way to circumvent this problem either by allowing local topological mesh changes or some kind of adaptive mesh refinement strategy. In this section, we will show how sensitive the FSI algorithm 


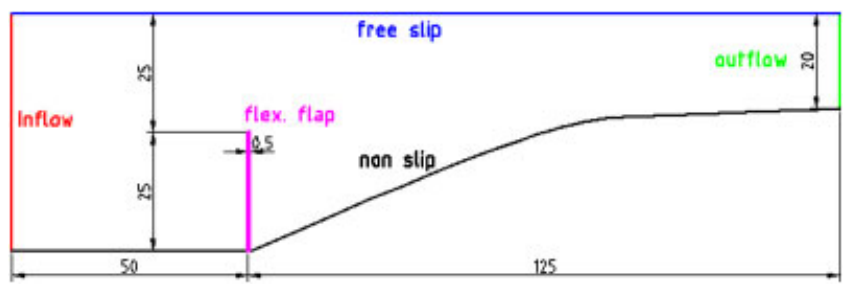

Figure 5. Problem description and dimensions $(\mathrm{cm})$.

is to mesh changes and whether it affects or not the convergence rate of the underlying numerical technique, a basic requirement for any coupling algorithm.

The test case chosen was first proposed by Neumann et al. in [4]. The sketch of the problem with dimensions and boundary conditions is depicted in Figure 5. The problem consists of a channel with a gradual contraction. Right before the contraction there is a flexible solid flap. The flap represents a rubber material with Young modulus $E=2.3 \times 10^{6} \mathrm{~N} / \mathrm{m}^{2}$, density $\rho_{\mathrm{s}}=1500 \mathrm{~kg} / \mathrm{m}^{3}$ and Poisson ratio $v=0.45$. The fluid is a silicon oil with density $\rho_{\mathrm{f}}=956 \mathrm{~kg} / \mathrm{m}^{3}$ and viscosity $\mu=0.145 \mathrm{~kg} /(\mathrm{ms})$. The fully developed laminar flow has a Reynolds number of $R e=100$.

The inflow velocity for the problem follows the law:

$$
V(t)= \begin{cases}\frac{V_{\max }}{2}\left[1-\cos \left(\frac{\pi t}{10}\right)\right] & \text { if } t \leqslant 10 \\ V_{\max } & \text { if } t>10\end{cases}
$$

where $V_{\max }=0.06067$ (Figure 6).

Four different mesh configurations were tested. Since the test case proposed has no analytical solution or experimental data, a reference solution with a highly refined mesh was taken as the exact solution. The mesh grading around the flap varies from 0.001 for the finest reference mesh to $0.002,0.004$ and 0.008 for the coarser. The meshes are shown in Figure 7. All fluid meshes match the solid mesh at the interface so that the solid mesh is also refined and the errors incurred in transferring loads and displacement are avoided.

Only the sensitivity with respect to the mesh size was studied. Thus, the time step size was kept constant and equal to $\Delta t=0.05$ for all the meshes and the error computations were carried out at steady state. This means that according to Equation (83) the value of $\beta$ will only change with respect to the mesh size. It is worth mentioning for $\beta=0$ (no interface Laplace matrix is used) the coupled problem does not converge in the very early stages of the solution (within the first five time steps).

The variable taken for the analysis was the flap tip deflection in the streamline direction corresponding to the $x$ coordinate at steady state. An $L_{2}$ norm was used to evaluate the error:

$$
L_{2}=\sqrt{\frac{1}{n} \sum_{i=1, n}\left|\mathbf{d}_{x}-\mathbf{r}_{x}\right|^{2}}
$$

where $\mathbf{r}_{x}$ is the reference displacement vector for the finer mesh. The steady state was reached at $t=25 \mathrm{~s}$ and the sum was performed for $\mathrm{d}_{x}$ and $\mathrm{r}_{x}$ between 25 and $35 \mathrm{~s}$, with $n=10 / \delta t$ being the total number of time steps. 


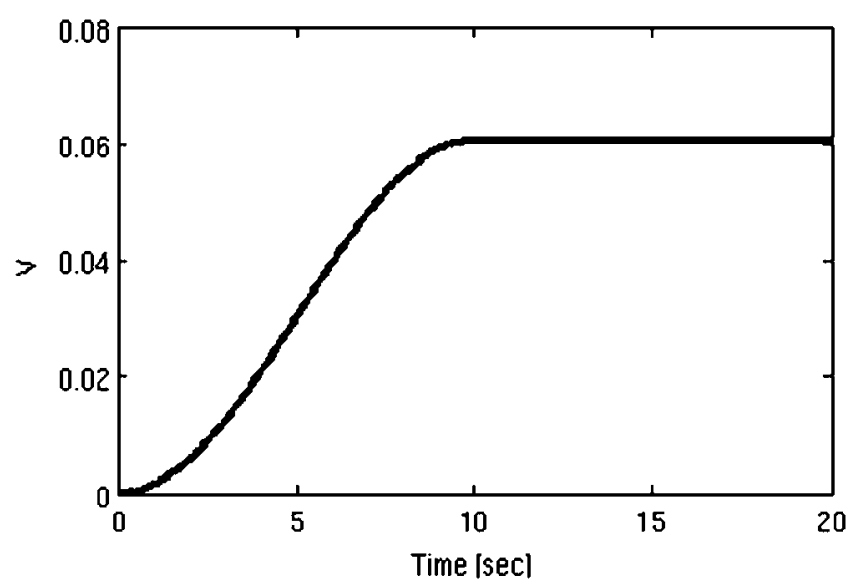

Figure 6. Fluid velocity.
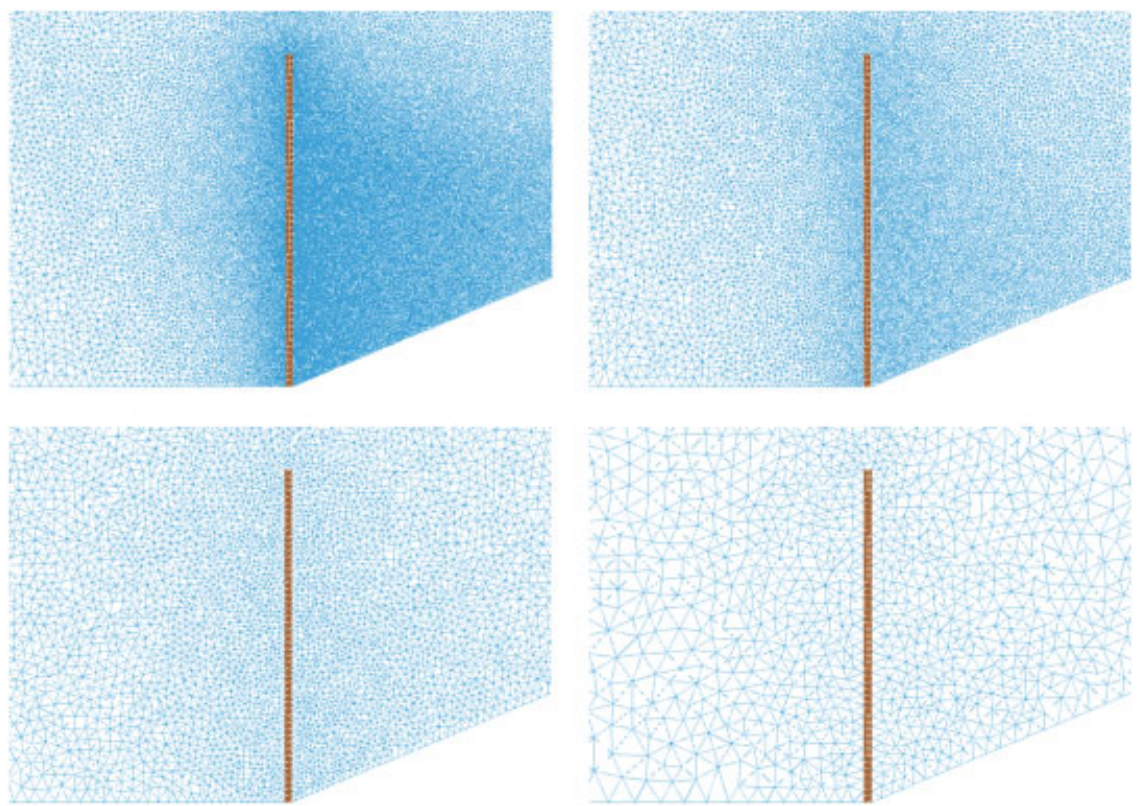

Figure 7. Different mesh densities used in the convergence analysis. Number of elements from finer to coarser: $101251,43838,16927$, and 4380 .

Figure 8 depicts the tip displacement with respect to time, which is in good agreement with the one shown in [4].

The error evaluated in each mesh is presented in a log-log plot in Figure 9 where the abscissa is the logarithmic of the error and the ordinate is the logarithmic of the mesh size. The curve 


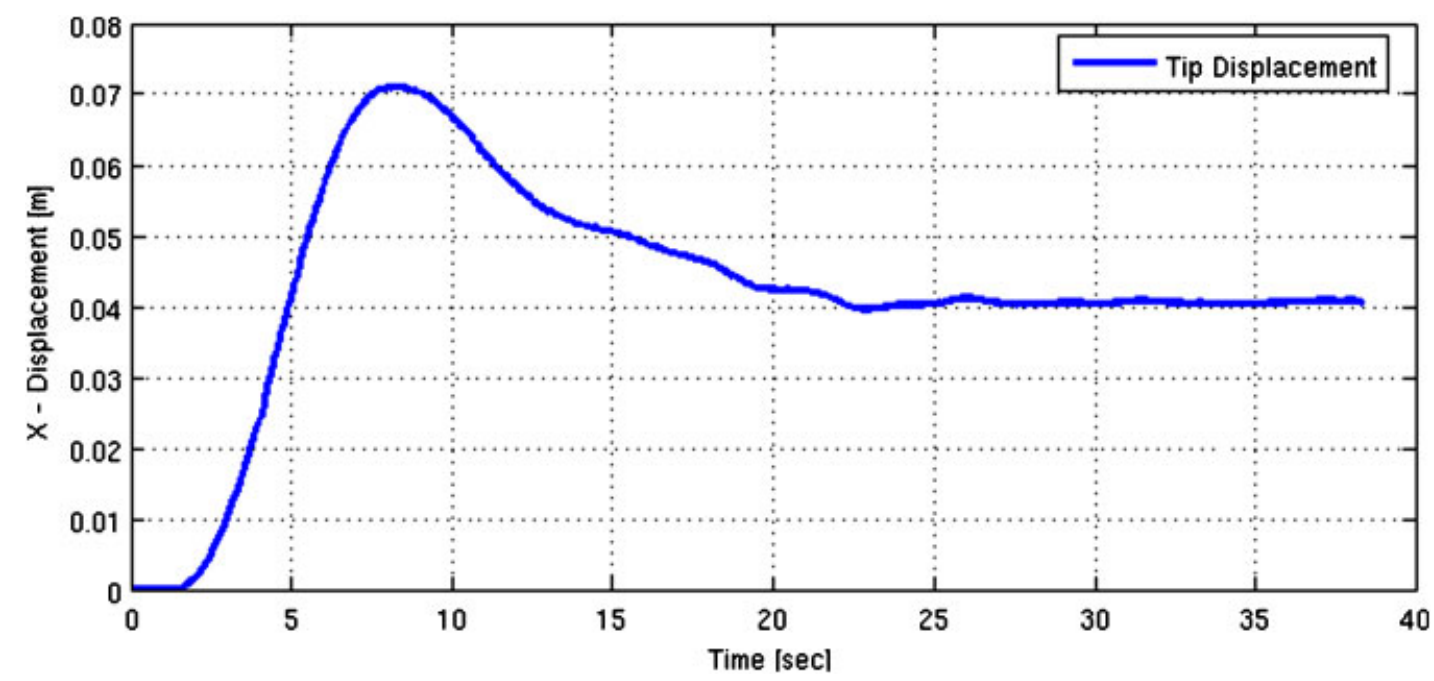

Figure 8. Tip deflection in the $x$ direction.

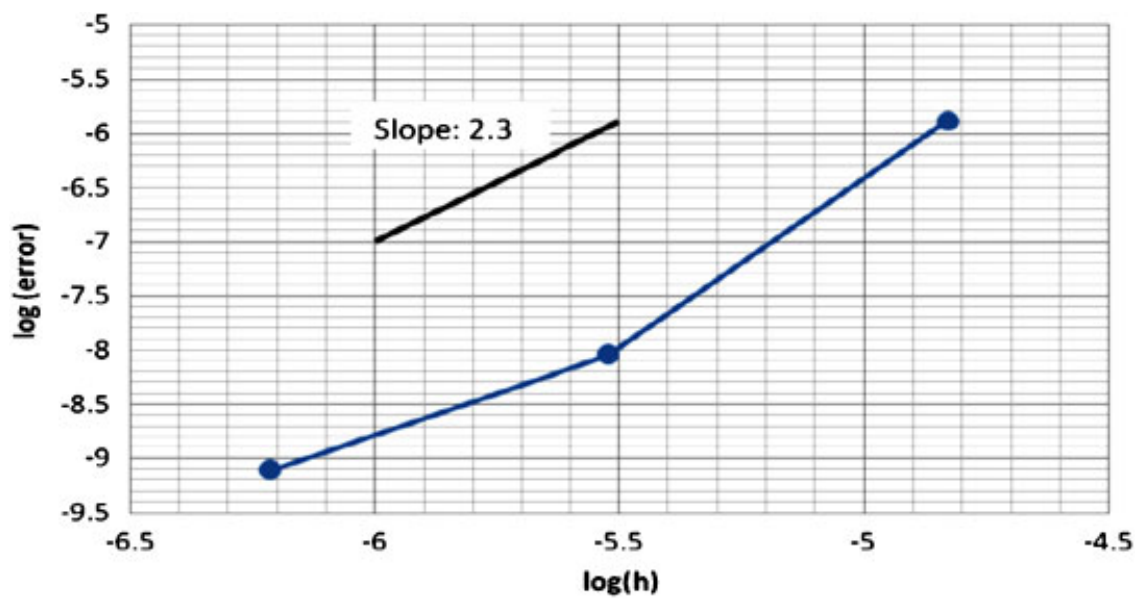

Figure 9. Convergence plot for the flexible flap problem.

slope represents the order of convergence. In this case an optimum quadratic convergence is found matching the individual order of convergence for the fluid and the solid formulation separately.

Figure 10 shows the flow vectors together with the velocity modulus. The first three time steps correspond to the initial deflection. At time $t=10 \mathrm{~s}$. The main vortex is detached and the maximum velocity is reached (see Figure 6). At this point the flap starts retracting until its steady-state position.

Finally, in Figure 11 we show the plot of the iteration number versus time for the intermediate mesh of size 0.004 . The iteration counts do not vary so much for the different meshes so only this case is shown. We can see an average value between 7-8 iterations per time step. This compares 

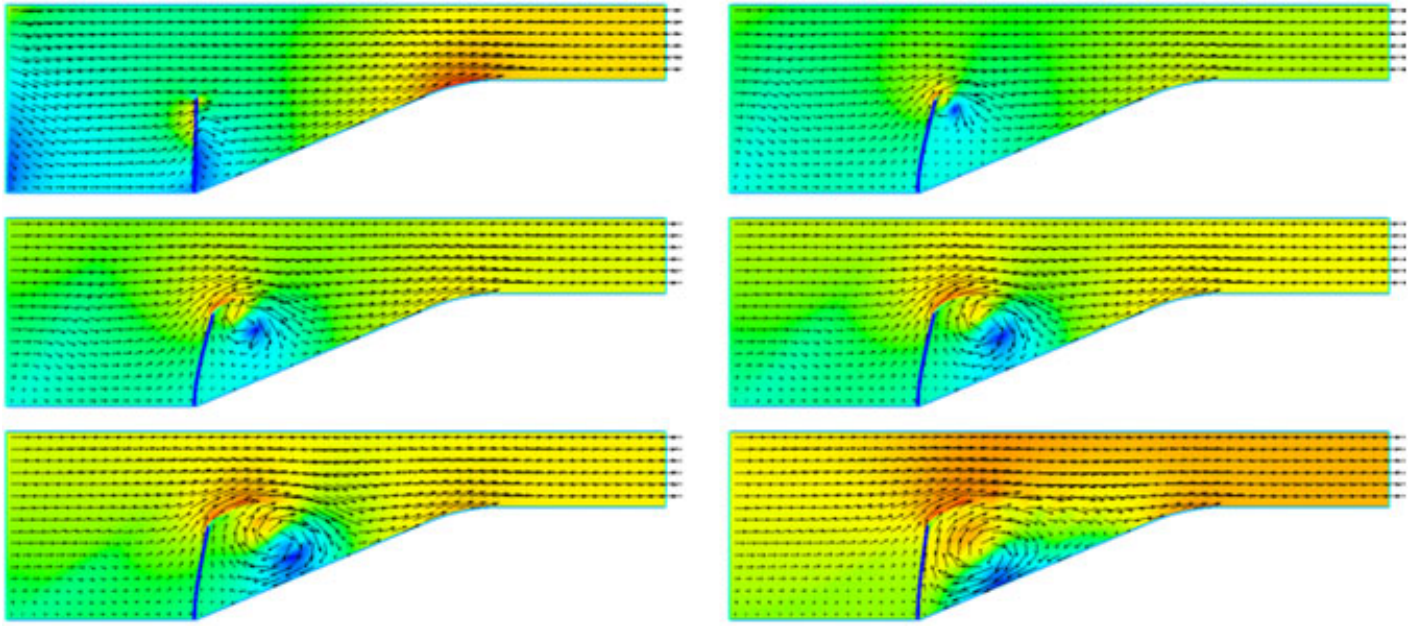

Figure 10. Time steps $2.5,5,10,12.5,15,25 \mathrm{~s}$.

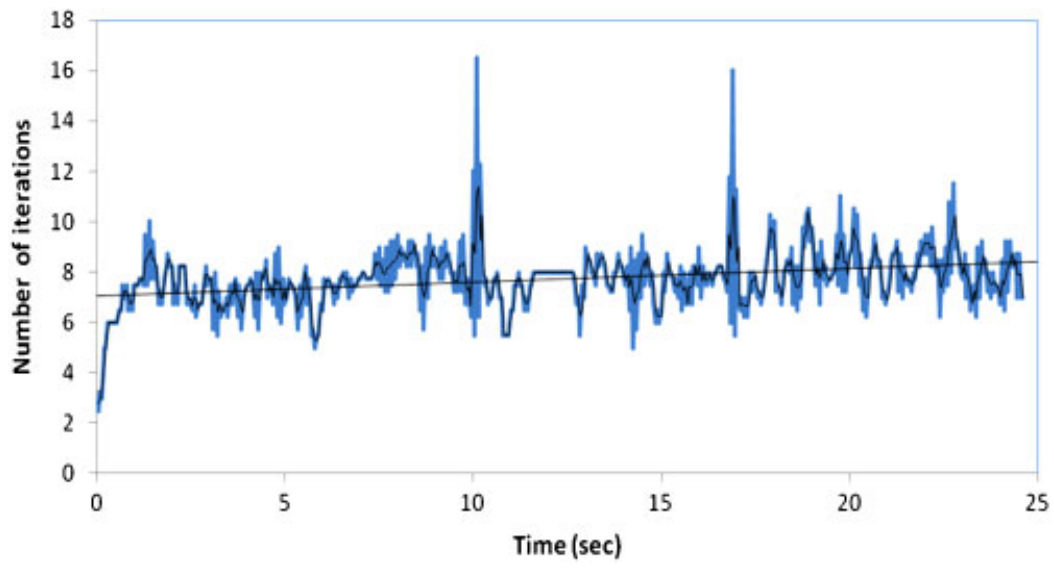

Figure 11. Number of iterations.

well with the most optimum algorithm proposed in [4]. The spikes in the iterations correspond to fluid features like vortex detachment that increase the non-linearity of the problem.

\subsection{Flexible flap $(3 D)$ in a converging channel}

The present example is a 3D version of the test case introduced in the previous section. In Figure 12 the 3D geometry is depicted and Figure 13 shows the surface mesh used in the solution process. The geometry is extruded in the $z$ direction but the flap is placed in the centre using a fraction of the width of the channel. In this way the flow is allowed to wrap around the flap. As no comparison is performed the Young modulus $E$ is modified to allow for larger displacements: $E=1.3 \times 10^{6} \mathrm{~N} / \mathrm{m}^{2}$. 

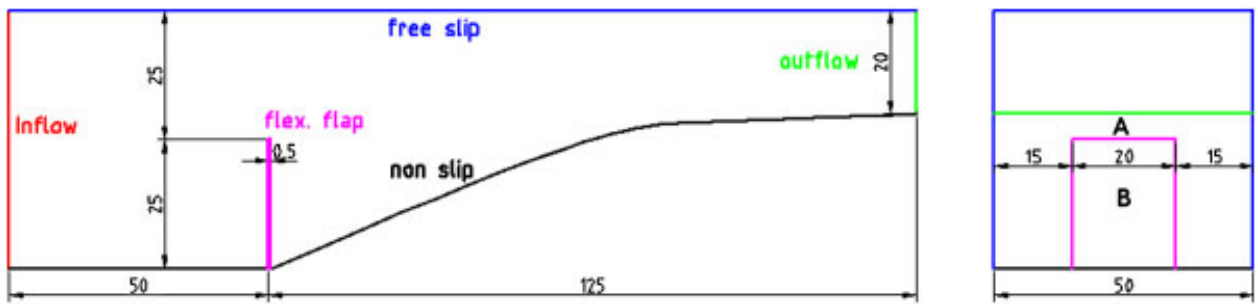

Figure 12. Problem description and dimensions $(\mathrm{cm})$.
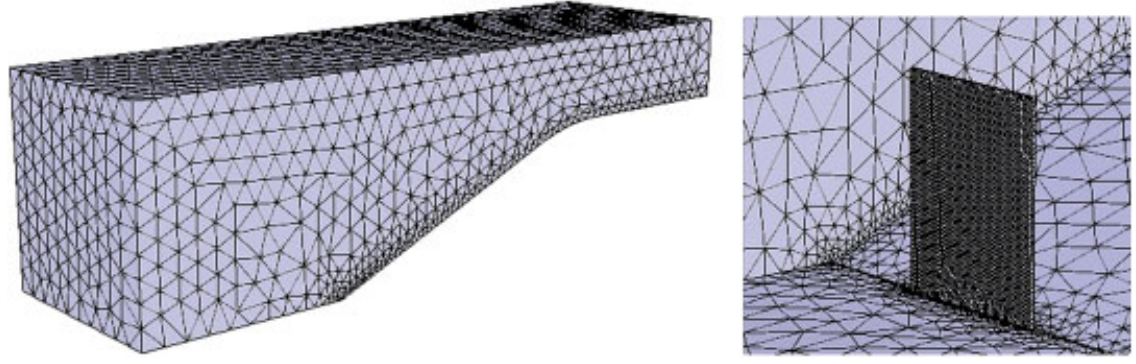

Figure 13. Left: the surface meshes for the full domain. Right: close-up of the flexible flap.

In Figure 14 the streamlines of velocity and the flap deflection are presented. Figure 15 shows the flap at its maximum deflection coloured with the values of Von Misses stresses. In the same figure the tip displacement is shown for points A and B (see Figure 11).

The large deformation of the flap triggered a re-meshing stage in the solver which was coupled to an error estimator. In this way each new mesh was enriched with a new element size with the objective of keeping the error constant throughout the mesh. As it was shown in the previous section the change in mesh size should not modify the convergence of the algorithm. Since the surface mesh size between the fluid and the solid structure does not change, the value of $\beta$ is kept constant throughout the whole problem. Figure 16 shows different element densities across the flap. A total of thirteen re-meshing stages were performed.

\subsection{Flexible valve in pulsatile flow}

The following example models the mechanics of a flexible valve immersed in a pulsatile flow. The valve opens and closes according to the flow rate in the channel. The objective of this test is to validate the model for a later application of the FSI algorithm proposed in real heart geometry. We realize that the heart solid mechanics is an extremely complex problem. The simplifications made in this test will allow us to evaluate the feasibility of applying the new algorithm to a more realistic approach. From the FSI point of view the complexity of the problem lays on the fact that heart tissue and blood flow have similar densities and therefore overcoming the difficulties of the added-mass effects is an important step for a successful FSI analysis of a real heart valve.

For the present analysis the fluid and solid physical properties resemble the properties of the real problem. The fluid density is $\rho_{\mathrm{f}}=1000 \mathrm{~kg} / \mathrm{m}^{3}$ and although the blood exhibits non-Newtonian 

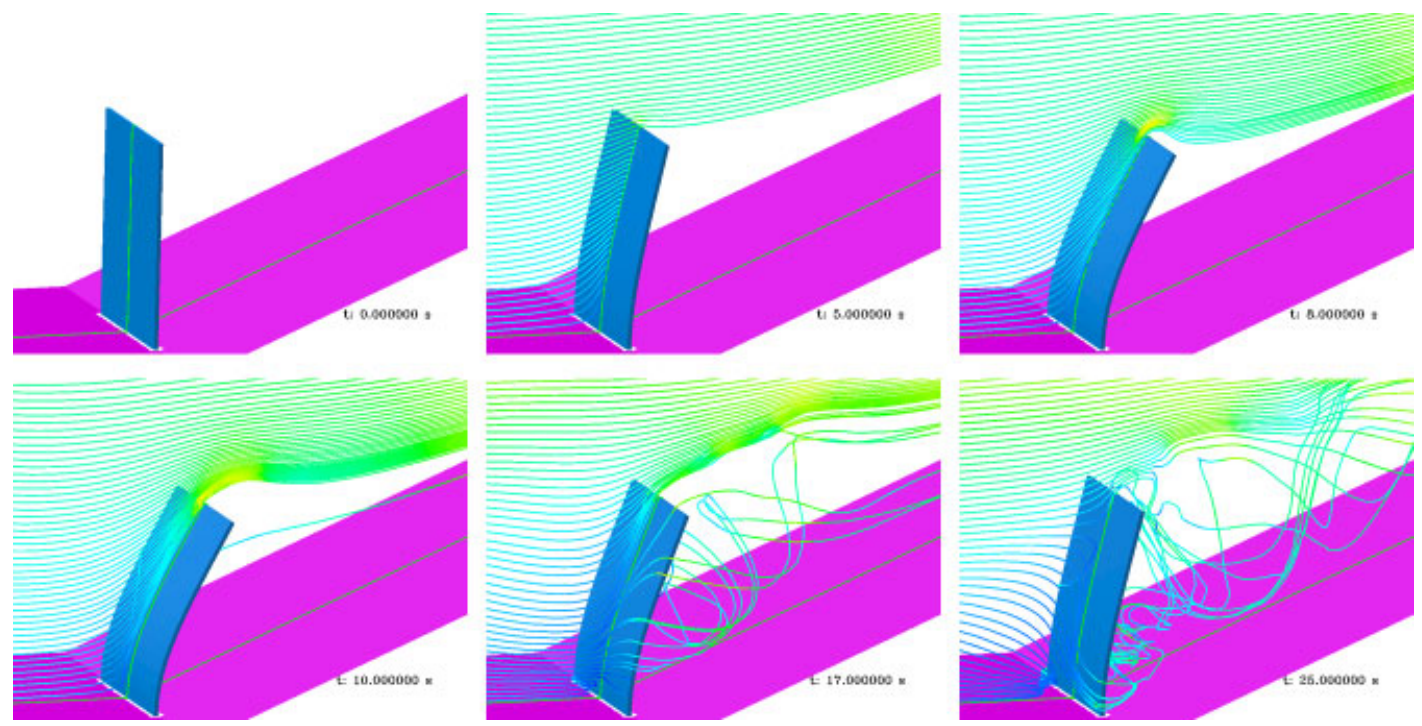

Figure 14. Streamlines of velocity for time steps $0,5,8,10,17$, and $25 \mathrm{~s}$.
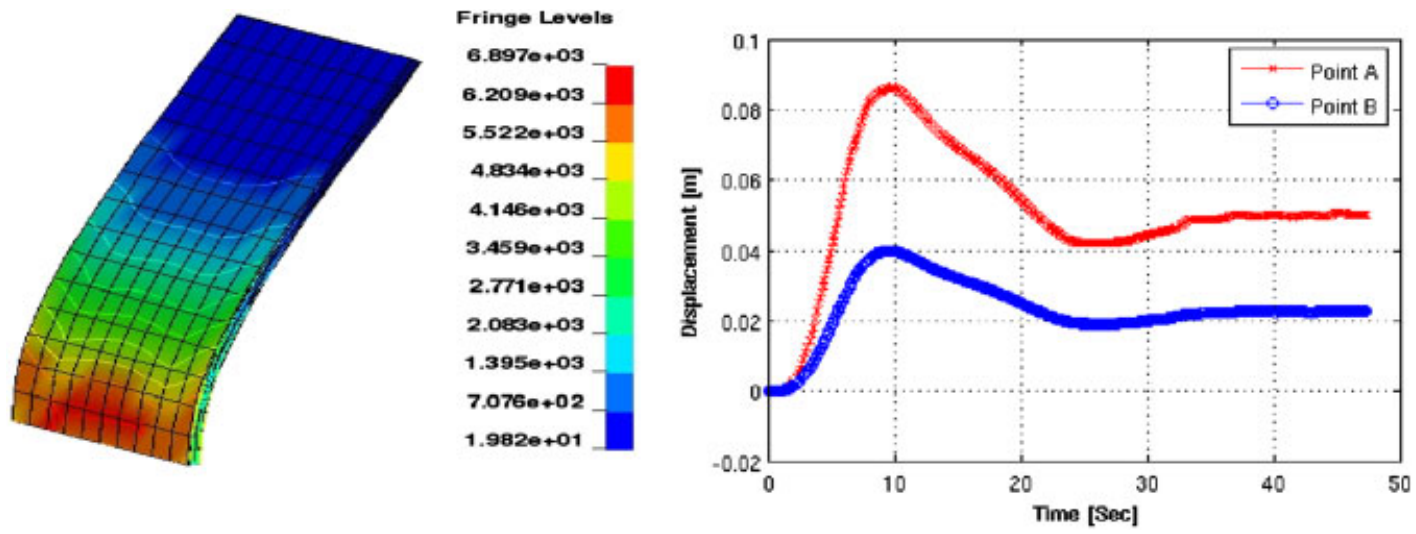

Figure 15. Von Mises stresses at maximum deflection and flap displacement as a function of time for points $\mathrm{A}$ and $\mathrm{B}$ indicated in Figure 12.

behaviour it is modelled here as a Newtonian flow with viscosity $\mu_{\mathrm{f}}=0.01 \mathrm{~kg} /(\mathrm{ms})$. We used an elastic solid to model the valve with $\rho_{\mathrm{s}}=1000 \mathrm{~kg} / \mathrm{m}^{3}, E=1.0 \times 10^{6} \mathrm{~N} / \mathrm{m}^{2}$ and $v=0.45$.

For this example the geometry is shown in Figure 17. Figure 18 shows the surface mesh used in the computation together with a close-up of the heart valve. The radius of the channel is $0.02 \mathrm{~m}$, which compares well with the average radius of the aorta of $0.012 \mathrm{~m}$. The average thickness of the left ventricle is $0.0011 \mathrm{~m}$ and the tricuspid valve thickness is $0.0005 \mathrm{~m}$ both in the order of our approximation that is $0.001 \mathrm{~m}$. The normal heart cycle at rest is $0.8 \mathrm{~s}$, which in our case was 

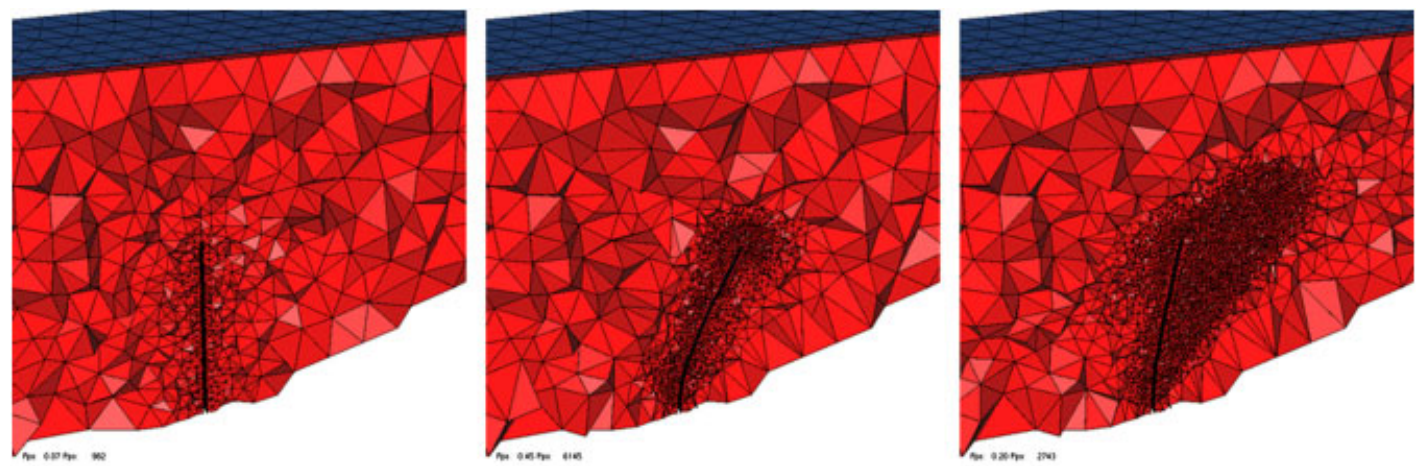

Figure 16. Tetrahedral mesh at different time steps.
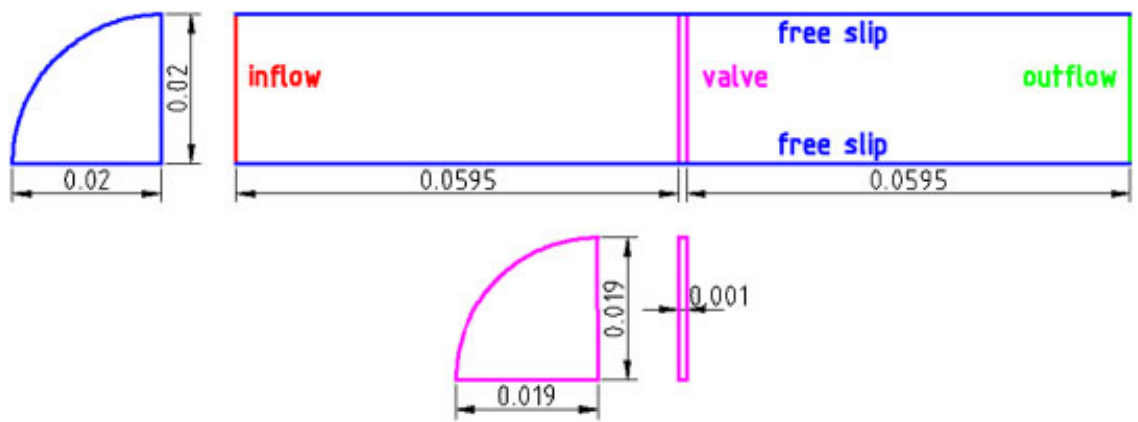

Figure 17. Geometry description for the flexible valve problem. Dimensions in meters.

represented by a cycle of approximate $0.4 \mathrm{~s}$. At the peak of the heartbeat the velocity in the Aorta for a person at rest may reach $0.75 \mathrm{~m} / \mathrm{s}$, about seven times larger than the one proposed in our experiment but close enough for a proof of concept case study.

The pulsatile flow is generated with the function:

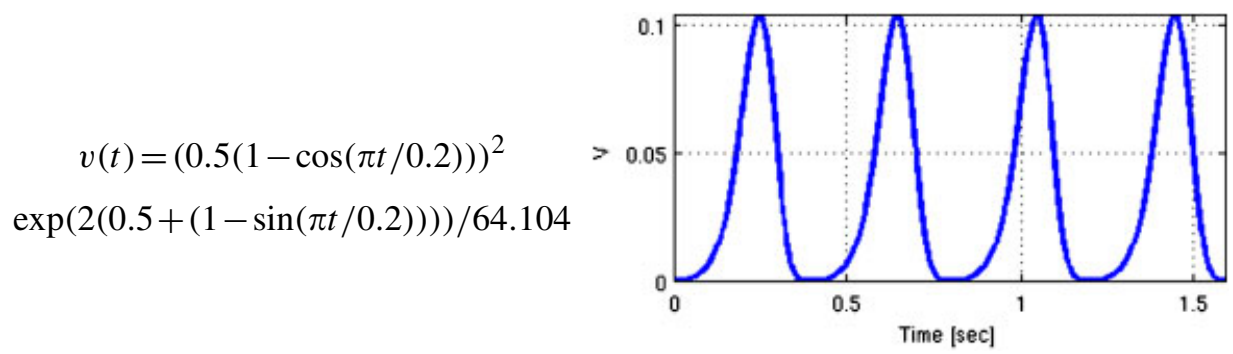

The problem was run for four complete cycles where the valve opens and closes once for each cycle. Owing to the large deformations of the valve several local as well as global re-meshing steps take place. 


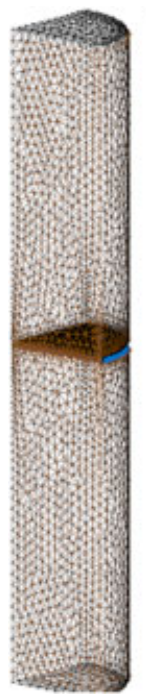

t: 0.000000 ह

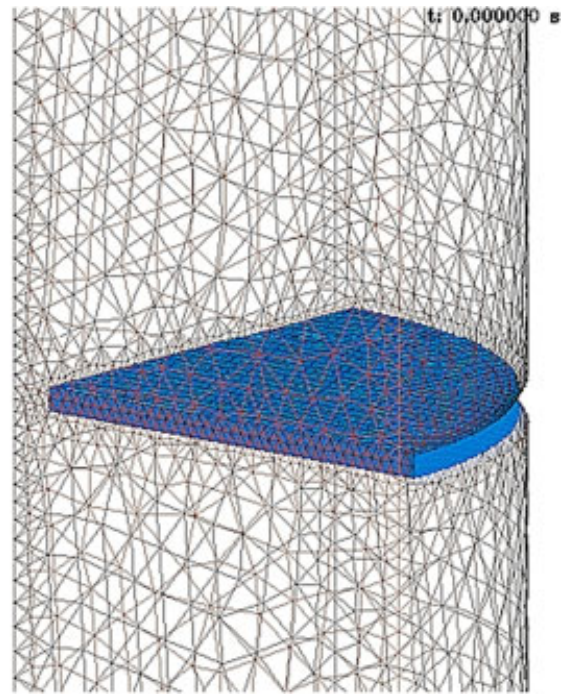

Figure 18. Surface mesh and valve close-up.
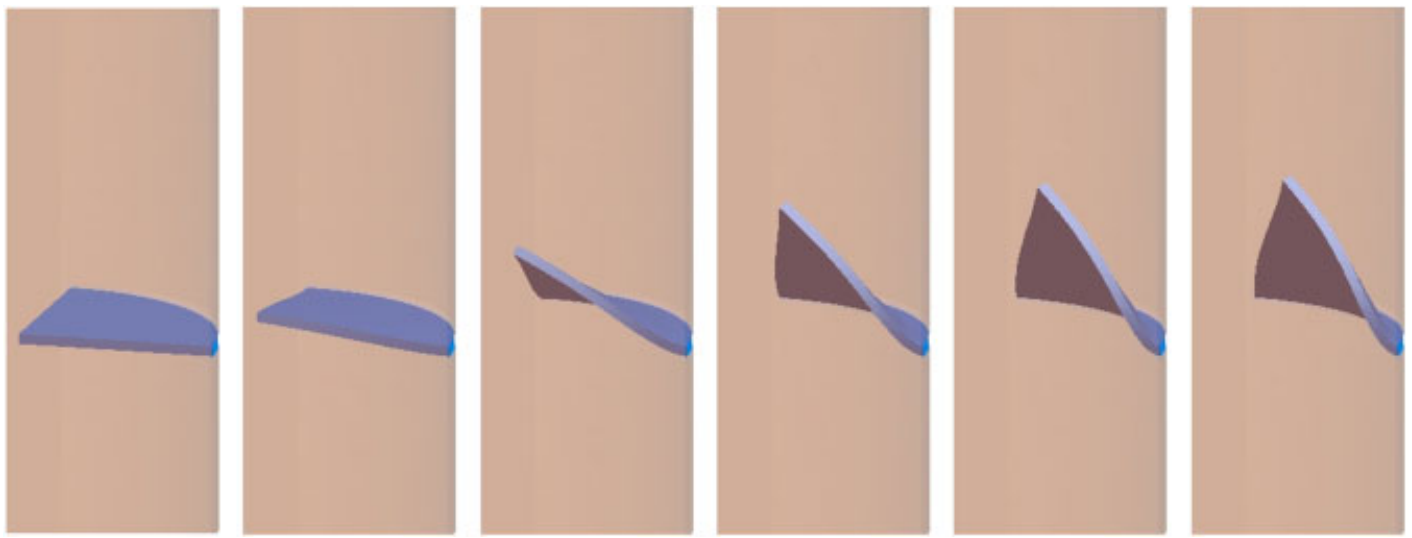

Figure 19. Valve deflection for time steps $t=0,0.016,0.2,0.22,0.24,0.28$.

The results are presented in Figure 19-22. First six different configurations of the valve are shown in Figure 19. The first frame represents the time of zero displacement and the last frame the point of maximum deflection.

Figure 20 shows a cut plane where the velocity vectors as well as the velocity modulus were projected.

In Figure 21 the projection of the pressure on the cut planes is shown. Finally, Figure 22 shows the total force transferred from the fluid to the value. 

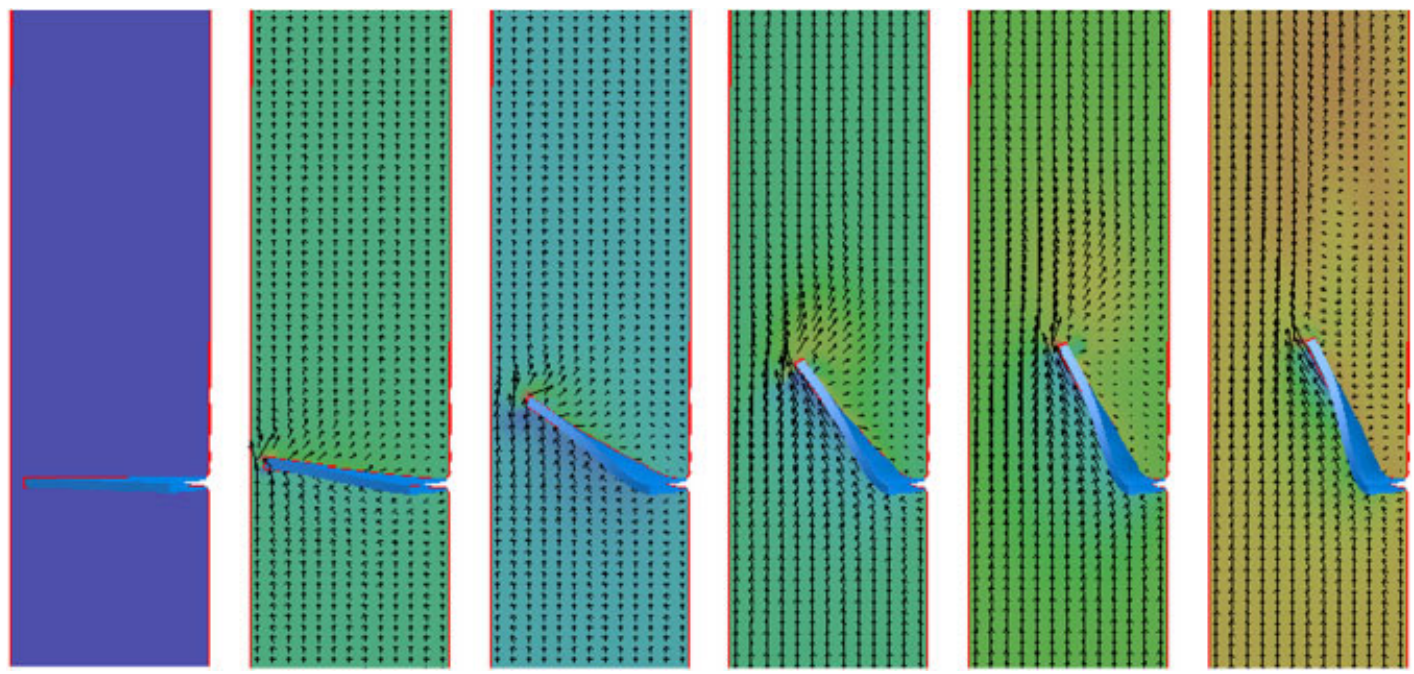

Figure 20. Cut plane showing velocity vectors for time steps $t=0,0.016,0.2,0.22,0.24,0.28$.
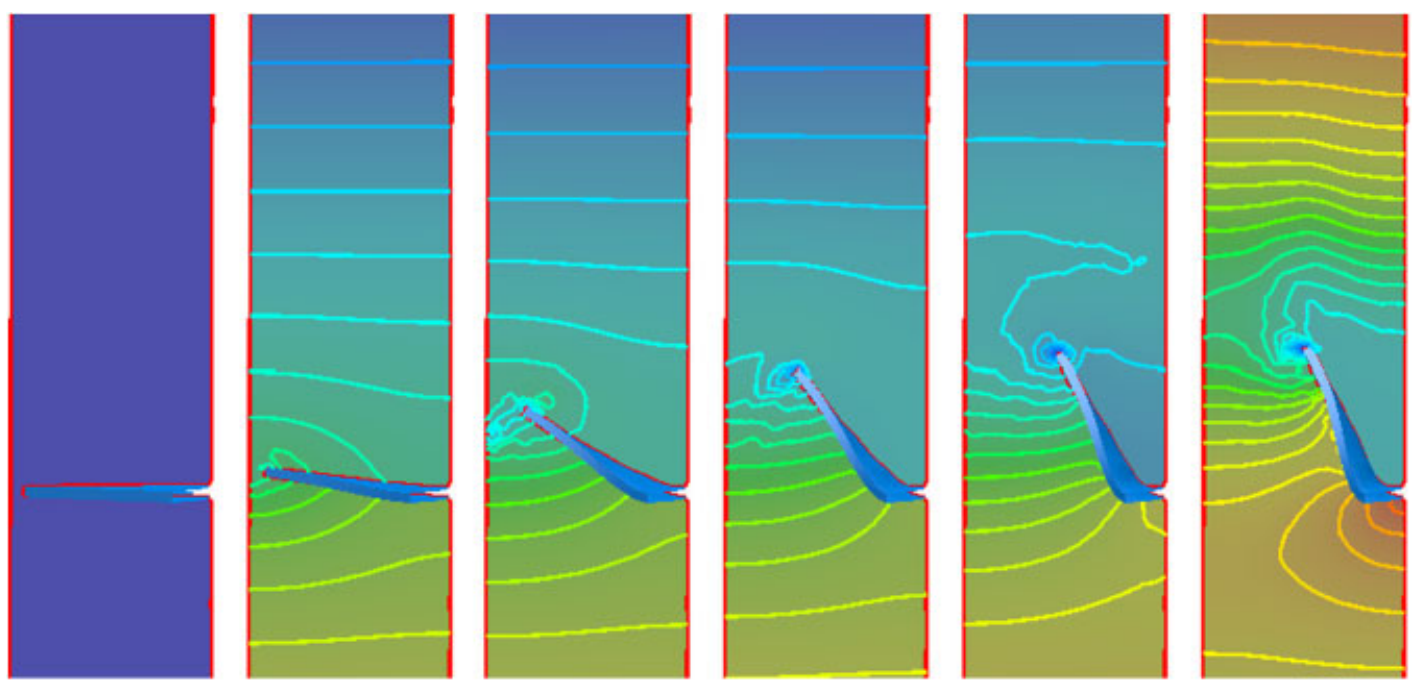

Figure 21. Cut plane with pressure iso-contours for time steps $t=0,0.016,0.2,0.22,0.24,0.28$.

\section{CONCLUSIONS}

One of the important key of the formulation presented is that it shows clearly how to segregate the pressure in the monolithic scheme in order to solve the FSI problems with a staggered algorithm.

Based on the pressure segregation scheme, we have also proposed an interface Laplace matrix that gives excellent convergence rates for the totality of the examples performed, even in those examples where the added-mass effect is important. 


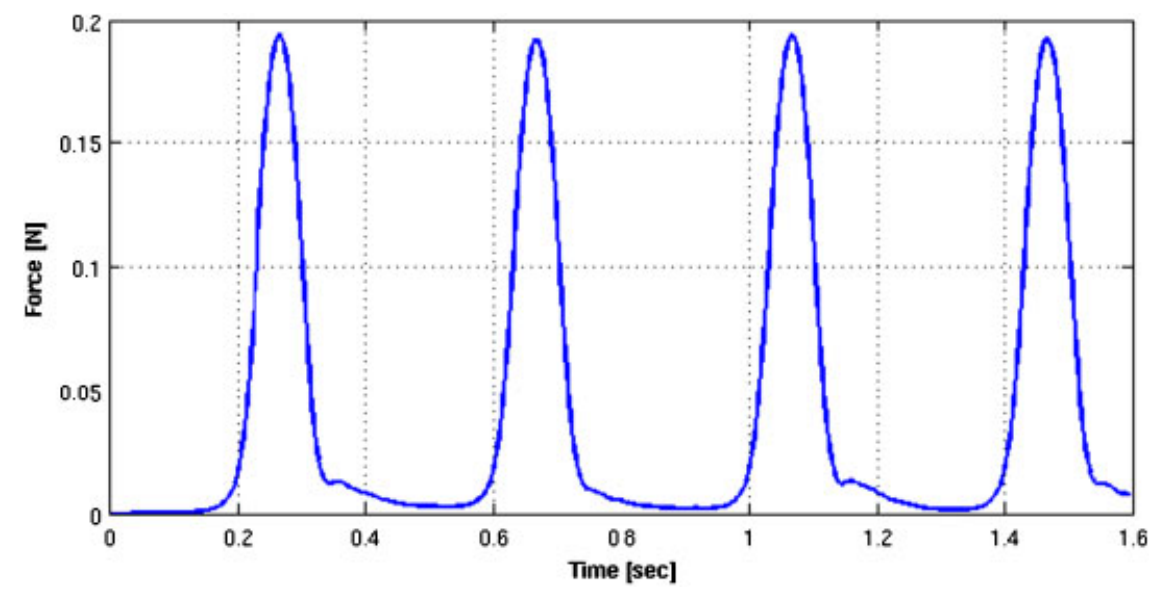

Figure 22. Total force transferred from the fluid to the valve as a function of time for the four complete cycles.

The pressure segregation method proposed for the solution of FSI problems with special emphasis in added-mass effects has shown an excellent behaviour with promising possibilities in the field of bio-medical applications.

The method was extended to strongly couple partitioned schemes with the same excellent results. This allows us to conclude that a correct understanding of the pressure segregation is the key issue to solve any FSI problem with a partitioned or a coupled scheme.

The correct understanding of the pressure segregation in the monolithic scheme allows us to perfectly predict the cases where staggered methods will present convergence problems. We can conclude that the added-mass effect is not only a problem of equal densities in the solid and the fluid. The non-convergence problem is also present in cases where the solid stiffness is low (soft materials) independently of the density rate. Moreover, a serious problem of staggered schemes, that is the divergence when the time step decreases, is also overcome using the new interface Laplace matrix.

The examples have shown that the partitioned approach preserves the convergence rate of each separate solver (fluid and solid), namely if both have quadratic approximation in space this rate is maintained after the coupling.

\section{ACKNOWLEDGEMENTS}

This work was partially supported by projects SEDUREC and XPRES of the 'Ministerio de Educación y Ciencia' of Spain.

\section{REFERENCES}

1. Felippa CA, Park KC. Staggered transient analysis procedures for coupled-field mechanical systems: formulation. Computer Methods in Applied Mechanics and Engineering 1980; 24:61-111.

2. Farhat C, Lesoinne M, Le Tallec P. Load and motion transfer algorithms for fluid/structure interaction problems with non-matching discrete interfaces: momentum and energy conservation, optimal discretization and application to aeroelasticity. Computer Methods in Applied Mechanics and Engineering 1998; 157:95-114. 
3. Piperno S, Farhat C. Partitioned procedures for the transient solution of coupled aeroelastic problems-part II. Computer Methods in Applied Mechanics and Engineering 2001; 190:3147-3170.

4. Neumann M, Tiyyagura SR, Wall WA, Ramm E. Robustness and efficiency aspects for computational fluid structure interaction. Computational Science and High Performance Computing II, vol. 91. Springer: Berlin, Heidelberg, 2006. ISSN: 1612-2909.

5. Lohner R, Yang C, Cebral J, Baum JD, Luo H, Pelessone D, Charman C. Fluid-structure interaction using a loose coupling algorithm and adaptive unstructured grids. AIAA-95-2259, 1995.

6. Rugonyi S, Bathe KJ. On the analysis of fully-coupled fluid flows with structural interactions-a coupling and condensation procedure. International Journal for Computational Civil and Structural Engineering 2000; 1:29-41.

7. Rugonyi S, Bathe KJ. On finite element analysis of fluid flows fully coupled with structural interactions. Computer Modeling and Simulation in Engineering 2001; 2:195-212.

8. Souli M, Ouahsine A, Lewin L. Arbitrary Lagrangian-Eulerian formulation for fluid-structure interaction problems. Computer Methods in Applied Mechanics and Engineering 2000; 190:659-675.

9. Le Tallec P, Mouro J. Fluid structure interaction with large structural displacements. Computer Methods in Applied Mechanics and Engineering 2001; 190:3039-3067.

10. Felippa CA, Park KC, Farhat C. Partitioned analysis of coupled mechanical systems. Computer Methods in Applied Mechanics and Engineering 2001; 190:3247-3270.

11. Walhorn E, Kolke A, Hubner B, Dinkler D. Fluid-structure coupling within a monolithic model involving free surface flows. Computers and Structures 2005; 83:2100-2111.

12. Dettmer W, Peric D. A computational framework for fluid-structure interaction: finite element formulation and applications. Computer Methods in Applied Mechanics and Engineering 2006; 195:5754-5779.

13. Causin P, Gerbeau J-F, Nobile F. Added-mass effect in the design of partitioned algorithms for fluid-structure problems. Computer Methods in Applied Mechanics and Engineering 2005; 194(42-44):4506-4527.

14. Badia S, Quaini A, Quarteroni A. Modular vs non-modular preconditioners for fluid-structure systems with large added-mass effect. Computer Methods in Applied Mechanics and Engineering 2008; 197(49-50):4216-4232.

15. Fernandez MA, Gerbeau JF, Grandmont C. A projection semi-implicit scheme for the coupling of an elastic structure with an incompressible fluid. International Journal for Numerical Methods in Engineering 2007; 69:794-821.

16. Forster Ch, Wall WA, Ramm E. Artificial added mass instabilities in sequential staggered coupling of nonlinear structures and incompressible viscous flows. Computer Methods in Applied Mechanics and Engineering 2007; 196:1278-1293.

17. Badia S, Nobile F, Vergara C. Fluid-structure partitioned procedures based on robin transmission conditions. Journal of Computational Physics 2008; 227:7027-7051.

18. Chorin AJ. Numerical solution of the Navier-Stokes equations. Mathematics of Computation 1968; 22:745-762.

19. Temam R. Sur l'approximation de la solution des _equations de Navier-Stokes par la methode des pas fractionaires (I). Archive for Rational Mechanics and Analysis 1969; 32:135-153.

20. Codina R. Pressure stability in fractional step finite element methods for incompressible flows. Journal of Computational Physics 2001; 170:112-140.

21. Oñate E, García J, Idelsohn SR, Del Pin F. FIC formulations for finite element analysis of incompressible flows Eulerian, ALE and Lagrangian approaches. Computer Methods in Applied Mechanics and Engineering 2006; 195:3001-3037.

22. Oñate E. Possibilities of finite calculus in computational mechanics. International Journal for Numerical Methods in Engineering 2004; 60(1):255-281.

23. Tezduyar TE, Mittal S, Ray SE, Shih R. Incompressible flow computations with stabilized bilinear and linear equal order interpolation velocity-pressure elements. Computer Methods in Applied Mechanics and Engineering 1992; 95:221-242.

24. Turek S. Efficient Solvers for Incompressible Flow Problems: An Algorithmic Approach in View of Computational Aspects. Lecture Notes in Computational Science and Engineering. Springer: Berlin, 1998.

25. Gerbeau JF, Vidrascu MA. A quasi-Newton algorithm based on a reduced model for fluid-structure interaction problems in blood flows. Mathematical Model and Numerical Analysis 2003; 37(4):631-648.

26. Del Pin F, Idelsohn SR, Oñate E, Aubry R. The ALE/Lagrangian particle finite element method: a new approach to computation of free-surface flows and fluid-object interactions. Computers and Fluids 2007; 36(1):27-38.

27. Idelsohn SR, Oñate E, Del Pin F, Calvo N. Fluid-structure interaction using the particle finite element method. Computer Methods in Applied Mechanics and Engineering 2006; 195:2100-2123. 